\title{
Equilibrium Conformations of Fluid Films with Bending Stiffness
}

\author{
Arnold Krawietz*,\#
}

Beuth Hochschule für Technik Berlin, Germany

\begin{abstract}
Minimal energy conformations of amphiphilic monolayers within a microemulsion are investigated. An exploitation of the Euler-Lagrange equations yields the internal forces and torques of the fluid film, which cannot be characterized by a simple surface tension. A numerical scheme reveals various properties of the equilibrium solutions of three families of bicontinuous structures. The influence of the constitutive behaviour is demonstrated not only on the basis of a quadratic bending energy density but also with a non-linear extension which exhibits symmetry breaking. The latter is essential to allow the three-phase coexistence of a microemulsion with excess phases of oil and water and hence the existence of an $\mathrm{X}$ point in the phase map.
\end{abstract}

Keywords: Amphiphile, curvature elasticity, bicontinuous structures, symmetry breaking, numerical scheme.

\section{INTRODUCTION}

A mixture of water, oil and amphiphile forms a microemulsion within which an amphiphilic monolayer separates an oil rich region from a water rich region ( $c f$. Strey [1], Gompper and Schick [2], and Safran [3, 4] for the experimental and theoretical background). Adopting the continuum mechanical approach, we regard this monomolecular fluid film of the amphiphilic surfactant as a curved sheet with bending stiffness. The curvature dependence of the bending energy density may be tuned e.g. by the temperature so that a variety of structures - with typical lengths in the nanometer range - can be observed experimentally: lamellae, micelles as well as bicontinuous surfaces. These impose various constraints on the enclosed water or oil volumes. Therefore the microemulsion of water, oil and amphiphile may coexist with excess phases of pure water and oil.

The goal of this paper is threefold:

\subsection{General Theory}

Starting from the postulate of minimal energy, we extract from the necessary Euler-Lagrangean differential equations the conditions of equilibrium of forces and moments and gain insight into the internal forces and torques of the fluid film. Since both membrane forces and transverse forces work together, the carrying behaviour differs markedly from that of a simple surface tension, which is familiar from the theory of capillarity.

\subsection{Computational Method}

We want to construct some equilibrium conformations. While the three structures with homogeneous curvature (lamella, sphere, and cylinder) can be treated exactly, a

*Address correspondence to this author at the Beuth Hochschule für Technik Berlin, Germany; Tel: 0049-30-7123267;

E-mail: krawietz@t-online.de

"Retired difference scheme is applied to handle bicontinuous surfaces. The latter divide space into two distinct, multiply-connected, intertwined subspaces. Such structures may be chaotic due to thermal fluctuations. But we restrict our attention to the static limit and discuss three ordered families which may be considered as periodic systems of tubes. The axes of the tubes are periodic graphs. (See Figs. $(\mathbf{1 , 2 , 3})$.) The plane graph of the first family, called tube3, is doubly-periodic. Three edges meet at each vertex, forming a Y-junction. Networks with such junctions are discussed in [5]. The spatial graphs of the other two families, called tube 4 and tube6, are triply-periodic with four or six edges meeting at each vertex. Prominent members of tube 4 and tube 6 are the minimal surfaces D (Diamond) and $\mathrm{P}$ (Primitive), respectively, of Schwarz [6, 7]. We restrict the admissible tubes by the postulate that any plane of symmetry of the skeletal graph is also a plane of symmetry of the tube. (Gyroids are not included in our treatment. They are triplyperiodic surfaces with three edges at each vertex - $c f$. [8] but have no planes of symmetry, which are essential for our numeric scheme).

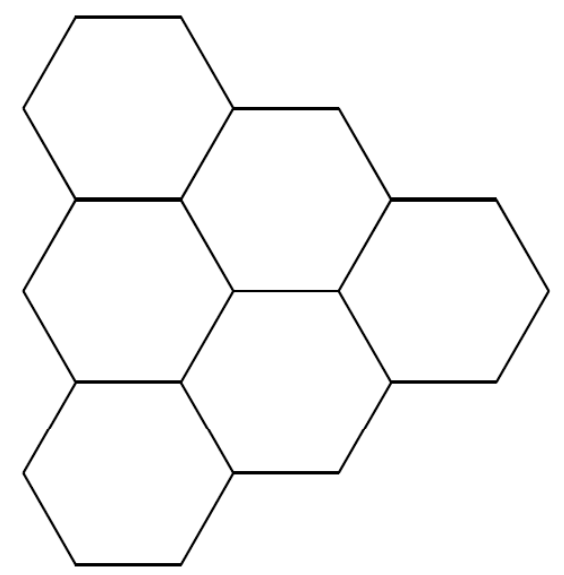

Fig. (1). A portion of the skeletal graph of tube3. 


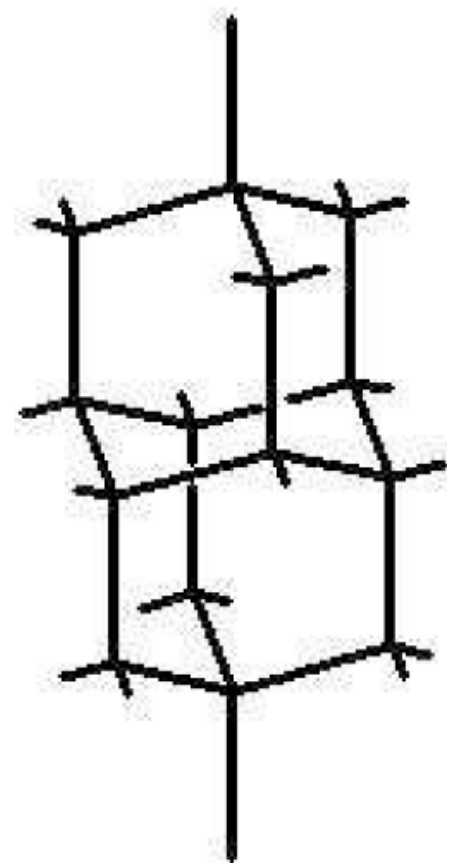

Fig. (2). A portion of the skeletal graph of tube4.

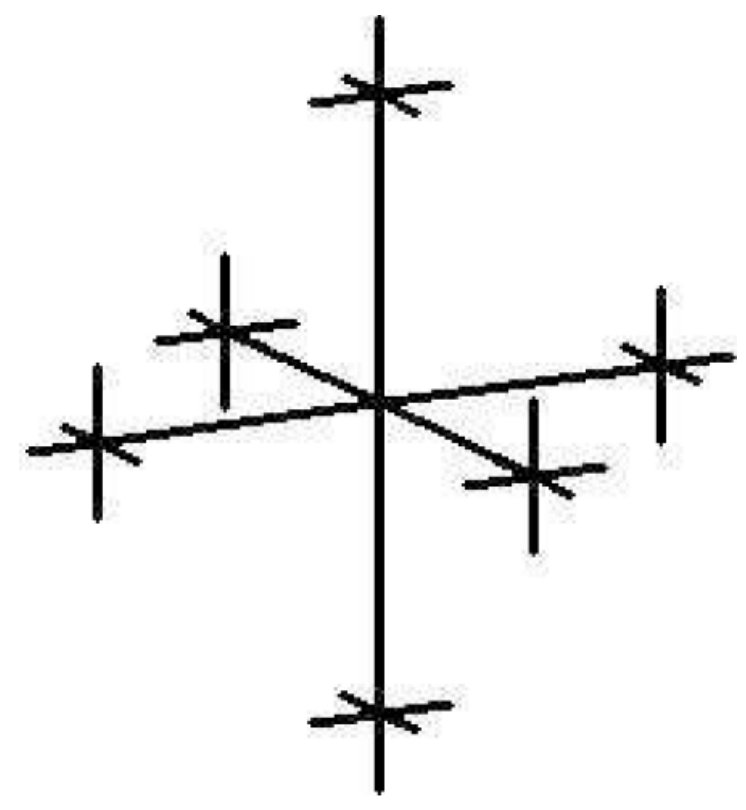

Fig. (3). A portion of the skeletal graph of tube6.

Let the distance $e$ of two neighbouring vertices be called the characteristic length of the structure. The skeletal graphs of tube 3 , tube 4 , tube 6 have periods of $3 e, 4 e$, and $e$, respectively in the direction of each edge. Let these graphs represent the axes of tubes filled with oil. In case of tube 4 and tube6, there exist also dual graphs, which represent the axes of tubes filled with water. They can be obtained by a translation of the original graphs; in case of tube 4 by $2 e$ in the direction of an edge, in case of tube 6 by $e / 2$ in each of the three directions of the edges. If the minimal surface D or $\mathrm{P}$, respectively, undergoes the same translation, then the oil and water volumes interchange their places.

\subsection{Study of the Constitutive Behaviour}

We investigate the influence of the constitutive law on the properties of the structures. If the energy density does not depend on the saddle-splay deformation, then the equilibrium solutions are surfaces of constant mean curvature. These have been studied extensively by Anderson et. al. [7] and were also applied to amphiphilic systems [8]. However, an influence of the saddle-splay deformation on the energy leads to different shapes. Some properties of these more complex surfaces are exploited in the paper in hand.

In this context, the effect of a symmetry breaking as discussed by Leitão et.al. [9] is of paramount importance. It could be demonstrated in [10] that such a symmetry breaking may explain the experimentally confirmed three-phase coexistence of a microemulsion with an oil and a water excess and allows the existence of an $\mathrm{X}$ point in the phase map, separating regions with none, one, and two excess phases.

Mathematical details are postponed into four appendices.

\section{THEORY}

We lay down the following suppositions:

- There is only one kind of structure; a coexistence of several structures is not treated.

- The monomeric solubility of the surfactant within oil or water is neglected.

- Oil and water are incompressible.

- The area of the middle surface of the surfactant film is constant.

- An environmental pressure is not considered.

A treatment without these restrictions can be found in the monograph [11].

The local curvature tensor of the surfactant film is written

$$
\mathbf{C}=c_{1} \mathbf{a}_{1} \otimes \mathbf{a}_{1}+c_{2} \mathbf{a}_{2} \otimes \mathbf{a}_{2}
$$

where $\otimes$ denotes dyadic multiplication, $\mathbf{a}_{1}$ and $\mathbf{a}_{2}$ are two orthogonal proper unit vectors and $c_{1}$ and $c_{2}$ the proper numbers of the tensor, called principal curvatures. The two invariants, trace and determinant, are given by

$$
\operatorname{tr} \mathbf{C}=2 H=c_{1}+c_{2}, \quad \operatorname{det} \mathbf{C}=K=c_{1} c_{2}
$$

and $H$ and $K$ are called mean and Gaussian curvature, respectively.

We assume the surfactant film to be endowed with a bending energy the density $w$ of which depends on the local curvature. Since a rigid rotation changes the vectors $\mathbf{a}_{1}$ and $\mathbf{a}_{2}$ but not the energy density, $w$ can only depend on the principal curvatures - or, equivalently, on the two invariants - and is thus an isotropic function of $\mathbf{C}$. If there is an oil and/or a water excess, then there will also be a plane surfactant film between the microemulsion and any excess 
phase. The energy density of these plane surfaces with area $A_{\mathrm{O}}$ or $A_{\mathrm{w}}$ shall be denoted by $w_{\mathrm{O}}$ or $w_{\mathrm{w}}$. The free energy of the system is therefore

$$
F=\int_{A} w(\mathbf{C}) d A+w_{\mathrm{O}} A_{\mathrm{O}}+w_{\mathrm{W}} A_{\mathrm{w}}
$$

The volume of the microemulsion is assumed to consist of a large number $N$ of cells, each of which contains the same bending energy, area of the film middle surface, oil volume, and water volume

$$
W_{C}=\int_{A_{C}} w(\mathbf{C}) d A, \quad A_{C}=\int_{A_{C}} d A, \quad V_{O C}, \quad V_{W C}
$$

Let the total volumes of oil and water of the mixture be denoted by $V_{\mathrm{O}}$ and $V_{\mathrm{w}}$. Then the following constraints must hold

$$
\begin{aligned}
& A-N A_{\mathrm{C}}-A_{\mathrm{O}}-A_{\mathrm{w}}=0 \\
& V_{\mathrm{O}}-N V_{\mathrm{OC}} \geq 0, \quad V_{\mathrm{w}}-N V_{\mathrm{wC}} \geq 0
\end{aligned}
$$

Strict inequality indicates that an excess is present.

We are interested in finding a conformation of the film that minimizes the energy under these constraints. Therefore, we multiply the constraints with Lagrangean parameters $f$, $p_{\mathrm{O}}, p_{\mathrm{w}}$, add them to the energy and finally divide by the constant value $A$. Introducing

$$
n=\frac{N}{A}, \quad L_{O}=\frac{V_{\mathrm{O}}}{A}, \quad L_{W}=\frac{V_{\mathrm{W}}}{A}
$$

called the number of cells per unit area of the film and oil length and water length of the mixture, respectively, we arrive at the expression

$$
\begin{aligned}
& E=n W_{\mathrm{C}}+w_{\mathrm{O}} \frac{A_{\mathrm{O}}}{A}+w_{\mathrm{w}} \frac{A_{\mathrm{w}}}{A} \\
& +f\left(1-n A_{\mathrm{C}}-\frac{A_{\mathrm{O}}}{A}-\frac{A_{\mathrm{W}}}{A}\right) \\
& \quad+p_{\mathrm{O}}\left(L_{O}-n V_{\mathrm{O} C}\right)+p_{\mathrm{w}}\left(L_{W}-n V_{\mathrm{wC}}\right)
\end{aligned}
$$

which we call the extended mean energy density. If an oil excess is present then we have to put $p_{\mathrm{O}}=0$, but $A_{\mathrm{O}} \neq 0$, otherwise $p_{\mathrm{O}} \neq 0$ and $A_{\mathrm{O}}=0$, and the same applies to the water.

Our search for the minimal free energy requires that $E$ be stationary with respect to any variation of the parameters $n, f, p_{\mathrm{O}}, p_{\mathrm{w}}$, and of the placement of the film, which influences $W_{\mathrm{C}}, A_{\mathrm{C}}, V_{\mathrm{OC}}$, and $V_{\mathrm{WC}}$. The vanishing of the partial derivative of $E$ with respect to $f, p_{\mathrm{O}}, p_{\mathrm{w}}$ yields the constraints - the latter two (with equality) only if there is no excess and hence $p_{\mathrm{O}} \neq 0$ and/or $p_{\mathrm{w}} \neq 0$. The vanishing of the derivative with respect to $n$ gives

$$
\begin{aligned}
& 0=W_{\mathrm{C}}-f A_{\mathrm{C}}-p_{\mathrm{O}} V_{\mathrm{OC}}-p_{\mathrm{w}} V_{\mathrm{wC}} \\
& \Rightarrow f=\bar{w}-p_{\mathrm{O}} l_{\mathrm{O}}-p_{\mathrm{w}} l_{\mathrm{w}}
\end{aligned}
$$

with the abbreviations

$$
\bar{w}=\frac{W_{\mathrm{C}}}{A_{\mathrm{C}}}, \quad l_{\mathrm{O}}=\frac{V_{\mathrm{OC}}}{A_{\mathrm{C}}}, \quad l_{\mathrm{w}}=\frac{V_{\mathrm{wC}}}{A_{\mathrm{C}}}
$$

which may be called mean energy density, oil length and water length of the cell.

The variation of the placement is elaborated in appendix B. (Notation: The dot product of vectors means the inner product. The dot and double dot products with tensors are defined by the rules $(\mathbf{b} \otimes \mathbf{c}) \cdot \mathbf{d}=\mathbf{b}(\mathbf{c} \cdot \mathbf{d})$, $(\mathbf{a} \otimes \mathbf{b}) \cdot(\mathbf{c} \otimes \mathbf{d})=(\mathbf{b} \cdot \mathbf{c}) \mathbf{a} \otimes \mathbf{d},(\mathbf{a} \otimes \mathbf{b}):(\mathbf{c} \otimes \mathbf{d})=(\mathbf{a} \cdot \mathbf{c})(\mathbf{b} \cdot \mathbf{d}))$. It is shown there that the internal torques and forces of the film can be characterized by the tensor of moments

$$
\begin{aligned}
& \mathbf{M}=-\frac{\partial w}{\partial \mathbf{C}}=-\frac{\partial w}{\partial c_{1}} \mathbf{a}_{1} \otimes \mathbf{a}_{1}-\frac{\partial w}{\partial c_{2}} \mathbf{a}_{2} \otimes \mathbf{a}_{2} \\
& =m_{1} \mathbf{a}_{1} \otimes \mathbf{a}_{1}+m_{2} \mathbf{a}_{2} \otimes \mathbf{a}_{2}
\end{aligned}
$$

the tensor of membrane forces

$$
\begin{aligned}
& \mathbf{T}=(w-f) \mathbf{1}_{\mathrm{T}}+\mathbf{C} \cdot \mathbf{M} \\
& =\left(w-f+c_{1} m_{1}\right) \mathbf{a}_{1} \otimes \mathbf{a}_{1}+\left(w-f+c_{2} m_{2}\right) \mathbf{a}_{2} \otimes \mathbf{a}_{2} \\
& =t_{1} \mathbf{a}_{1} \otimes \mathbf{a}_{1}+t_{2} \mathbf{a}_{2} \otimes \mathbf{a}_{2}
\end{aligned}
$$

- where $\mathbf{1}_{\mathrm{T}}$ denotes the unit tensor in the tangential plane - and an operator of transverse forces

$$
\mathbf{q}_{\mathrm{T}}=q_{1} \mathbf{a}_{1}+q_{2} \mathbf{a}_{2}
$$

which is obtained from

$$
\mathbf{q}_{\mathrm{T}}=\mathbf{1}_{\mathrm{T}} \cdot\left(\mathbf{M} \cdot \nabla_{\mathrm{T}}\right)
$$

as the tangential part of the divergence of the moment tensor field. The last equation expresses the equilibrium of moments, while the equilibrium of forces normal to the film is given by

$$
\mathbf{T}: \mathbf{C}+\mathbf{q}_{\mathrm{T}} \cdot \nabla_{\mathrm{T}}=-p_{n}
$$

with $-h$ denotes the constant thickness of the film -

$$
\begin{aligned}
p_{n}= & p_{\mathrm{w}}\left(1+\operatorname{tr} \mathbf{C} \frac{h}{2}+\operatorname{det} \mathbf{C} \frac{h^{2}}{4}\right) \\
& -p_{\mathrm{O}}\left(1-\operatorname{tr} \mathbf{C} \frac{h}{2}+\operatorname{det} \mathbf{C} \frac{h^{2}}{4}\right)
\end{aligned}
$$

which enables the interpretation of $p_{\mathrm{O}}$ and $p_{\mathrm{w}}$ as the pressures within the oil and water cavities and of $p_{n}$ as the effective local pressure acting on the middle surface in the direction of the unit normal $\mathbf{n}$, which points towards the oil.

Actually, a mixture needs some time to reach a state of minimal energy. During that time, the position of the test tube and, if excesses have formed, also the values of $A_{0}$ and $A_{\mathrm{w}}$ are held constant. After the state of equilibrium is reached, let us tilt the test tube so that these areas increase by $\dot{A}_{\mathrm{O}}$ and $\dot{A}_{\mathrm{w}}$. The mean energy density of the system then increases by 


$$
\dot{E}=\dot{E}_{0}+\frac{1}{A}\left[\left(w_{\mathrm{O}}-f\right) \dot{A}_{\mathrm{O}}+\left(w_{\mathrm{W}}-f\right) \dot{A}_{\mathrm{w}}\right]
$$

where $\dot{E}_{0}$ denotes that part of the increment which is due to changes of $f, p_{\mathrm{O}}, p_{\mathrm{w}}, n$ and the placement. But that part is zero because we start from a state of equilibrium. So the increment of the free energy of the system is seen to be equal to the power of surface tensions

$$
\dot{F}=A \dot{E}=t_{\mathrm{O}} \dot{A}_{\mathrm{O}}+t_{\mathrm{W}} \dot{A}_{\mathrm{W}}
$$

with

$$
t_{\mathrm{O}}=w_{\mathrm{O}}-f, \quad t_{\mathrm{w}}=w_{\mathrm{w}}-f
$$

The isotropic tensor of membrane forces becomes

$$
\mathbf{T}_{\mathrm{O}}=\left(w_{\mathrm{O}}-f\right) \mathbf{1}_{\mathrm{T}}, \quad \mathbf{T}_{\mathrm{w}}=\left(w_{\mathrm{w}}-f\right) \mathbf{1}_{\mathrm{T}}
$$

Since $\mathbf{C}=\mathbf{0}$ in the phase boundaries, this representation is in full accord with that of the membrane forces of the curved film, except that the energy density $w$ is replaced by $w_{\mathrm{O}}$ or $w_{\mathrm{W}}$. The simplest assumption would be $w_{\mathrm{O}}=w_{\mathrm{W}}=w(\mathbf{C}=\mathbf{0})$, in which case the bending moments in that plane surface are $\mathbf{M}=-\partial w / \partial \mathbf{C}(\mathbf{C}=\mathbf{0})$.

The role of the phase boundaries is now clarified, and we will neglect their contributions in all our computations, thus assuming $A_{\mathrm{O}}=0, A_{\mathrm{W}}=0$ even if there are excesses.

The behaviour that we found contrasts with the theory of capillarity. There we have a surface energy density $w_{0}=$ const and an isotropic surface tension $\mathbf{T}=w_{0} \mathbf{1}_{\mathrm{T}}$ even with curved surfaces, but no transverse forces and no moments.

\section{Finding 1}

The internal forces and torques of our fluid film are by far more copious than those of the theory of capillarity and are identical to those of a solid shell ( $c f$. Flügge [12]). Generally, the tensor of membrane forces $\mathbf{T}$ is not isotropic $\left(t_{1} \neq t_{2}\right)$, and we have not only normal $\mathbf{e} \cdot \mathbf{T} \cdot \mathbf{e}$ but also shearing forces $\mathbf{g} \cdot \mathbf{T} \cdot \mathbf{e} .(\{\mathbf{e}, \mathbf{g}\}$ denotes an orthonormal basis in the tangential plane which does not coincide with the basis $\left\{\mathbf{a}_{1}, \mathbf{a}_{2}\right\}$ of proper vectors). In addition, there is a tensor of moments $\mathbf{M}$, the components of which can be interpreted as bending and twisting moments $\mathbf{e} \cdot \mathbf{M} \cdot \mathbf{e}$ and $\mathbf{g} \cdot \mathbf{M} \cdot \mathbf{e}$, respectively. If the tangential part of the divergence of $\mathbf{M}$ is not zero then the equilibrium of moments additionally requires the existence of transverse internal forces, described by the operator $\mathbf{q}_{\mathrm{T}}$. The existence of all these internal forces and torques in a fluid film was already pointed out by Helfrich [13].

\section{Finding 2}

We identify two totally different mechanisms which carry the surface pressure $p_{n}$ : The change of the direction of membrane forces due to curvatures $\left(\mathbf{T}: \mathbf{C}=t_{1} c_{1}+t_{2} c_{2}\right)$ and the divergence of transverse forces $\left(\mathbf{q}_{\mathrm{T}} \cdot \nabla_{\mathrm{T}}\right)$, the latter being the only mechanism possible even in a plane plate.

\section{Finding 3}

The carrying contribution of the membrane forces can be further elucidated. To this purpose, we eliminate $f$, which may be interpreted as a chemical potential, from $\mathbf{T}$ by means of (2) and arrive at

$$
\mathbf{T}=\left(w-\bar{w}+p_{\mathrm{O}} l_{\mathrm{O}}+p_{\mathrm{w}} l_{\mathrm{w}}\right) \mathbf{1}_{\mathrm{T}}+\mathbf{C} \cdot \mathbf{M}
$$

and

$$
\mathbf{T}: \mathbf{C}=(w-\bar{w}) \operatorname{tr} \mathbf{C}+\left(p_{\mathrm{o}} l_{\mathrm{O}}+p_{\mathrm{w}} l_{\mathrm{w}}\right) \operatorname{tr} \mathbf{C}+\mathbf{M}(\mathbf{C}): \mathbf{C}^{2}
$$

The first term is present if the local value $w$ of the energy density differs from the mean value $\bar{w}$; it is therefore absent with spheres and cylinders. The second term arises if there is a constraint on the oil or the water volume so that pressures $p_{\mathrm{O}} \neq 0$ and/or $p_{\mathrm{W}} \neq 0$ exist, and the third is due to the presence of internal moments in the film. While the first and the second term are influenced by properties of the structure as a whole, the third term only depends on the local curvature.

\section{Finding 4}

If we let the film thickness $h \rightarrow 0$ and apply the theory of capillarity, then the equilibrium condition of forces (4) with (5) reduces to the membrane equation

$$
\begin{aligned}
& \mathbf{T}: \mathbf{C}=w_{0} \operatorname{tr} \mathbf{C}=-p_{n}=p_{\mathrm{O}}-p_{\mathrm{w}} \\
& \Rightarrow \operatorname{tr} \mathbf{C}=2 H=\frac{1}{w_{0}}\left(p_{\mathrm{O}}-p_{\mathrm{w}}\right)=\text { const }
\end{aligned}
$$

So the placement of the surface must be one with constant mean curvature. Such a special surface appears in the case of our fluid film only if no saddle-splay stiffness exists. But then we have $w=0$ and $p_{\mathrm{O}}=p_{\mathrm{w}}=0$ while $H \neq 0$ is possible, as will be demonstrated below. It is therefore erroneous to assume that the mean curvature of an interface always represents a difference in pressure.

\section{Finding 5}

The surface tension in the plane phase boundaries must not be confused with the internal forces within the curved fluid film. If there is an oil excess and a water excess, then (6) with (2) yields

$$
t_{\mathrm{O}}=w_{\mathrm{O}}-\bar{w}, \quad t_{\mathrm{w}}=w_{\mathrm{w}}-\bar{w}
$$

This has been pointed out by Strey ([1], eq. (14)). The energy terms on the right-hand sides may become nearly equal under suitable environmental conditions, e.g. temperature, so that the surface tensions $t_{\mathrm{O}}$ or $t_{\mathrm{w}}$ may assume ultralow values near zero. This is an essential effect of the presence of a surfactant in the phase boundary. If there is an oil excess but no water excess, then we have

$$
t_{\mathrm{O}}=w_{\mathrm{O}}-\bar{w}+p_{\mathrm{w}} l_{\mathrm{w}}
$$


with tube4 and tube6. In case of sphere, cylinder or tube3, however, we assume that there is enough water to prevent a contact between the single structures. The last equation is then valid with $p_{\mathrm{W}}=0$.

\section{Finding 6}

The simultaneous addition of a constant value to the energy densities $w, w_{\mathrm{O}}, w_{\mathrm{W}}$ has no influence on the internal forces and moments in the curved film and in the plane phase boundaries, since only differences and derivatives enter the forces and moments, respectively. This is in contrast to the theory of capillarity, where energy density and membrane forces are identical.

\section{Finding 7}

If the energy density of the film actually does not depend on the curvature but is constant then our search for a conformation with minimal energy is vacuous. Note that the area of the film is assumed to be fixed and so is the total energy which is hence the same for all conformations. On the other hand, the energy density of a surface is assumed constant in the theory of capillarity, too. Nevertheless, the search for a conformation with minimal energy is meaningful there since the area of the surface is not restricted.

\section{Finding 8}

Both analytical and numerical computations reveal that the pressures within the oil and water cavities have negative sign. This is plausible: If there is not enough oil or water to form an excess, then it must contract the cavities. Nevertheless, the fluids will not tear since the cavities are extremely small and the formation of free surfaces would require too much energy.

\section{EXACT SOLUTIONS}

\subsection{Sphere}

We consider spheres which enclose oil; $R=1 / c$ is the radius of the middle surface of the film and $h$ its thickness. We identify one sphere with a cell and consider first the case without an oil excess. The extended mean energy density is then

$$
\begin{aligned}
& E=n w(c) \frac{4 \pi}{c^{2}}+f\left(1-n \frac{4 \pi}{c^{2}}\right) \\
& +p_{\mathrm{O}}\left[L_{\mathrm{O}}-n \frac{4}{3} \pi\left(\frac{1}{c}-\frac{h}{2}\right)^{3}\right]
\end{aligned}
$$

The derivatives with respect to $p_{\mathrm{O}}$ and $f$ must vanish. This yields the following two equations for the determination of $c$ and $n$.

$$
\left(1-\frac{h}{2} c\right)^{3}-3 L_{\mathrm{O}} c=0, \quad n=\frac{c^{2}}{4 \pi}
$$

Next, we study the vanishing of the derivatives with respect to $n$ and $c$ and note $d w / d c=\partial w / \partial c_{1}+\partial w / \partial c_{2}=-m_{1}-m_{2}=-2 m$. We find

$$
\begin{aligned}
& p_{\mathrm{O}}=3 c\left(1-\frac{h}{2} c\right)^{-3}(w-f) \\
& =2 c\left(1-\frac{h}{2} c\right)^{-2}(w-f+m c)
\end{aligned}
$$

This implies

$$
\begin{aligned}
& w-f=\frac{2-h c}{1+h c} m c \\
& t=w-f+m c=\frac{3}{1+h c} m c
\end{aligned}
$$

and

$$
p_{\mathrm{O}}=\frac{24 m c^{2}}{(2-h c)^{2}(1+h c)}
$$

If an oil excess exists, then $c$ cannot be computed from the volume constraint. Instead, we make use of the statement $p_{\mathrm{O}}=0$ and find - because of $c \neq 0$ -

$$
w-f=0, \quad m=0, \quad t=0
$$

So the sphere is free of internal forces and torques, and the curvature $c$ is now obtained from the evident condition $-2 m=d w(c) / d c=0$.

\subsection{Cylinder}

We consider cylinders with radius $R=1 / c=1 / c_{1}$ which enclose oil. We identify a piece of one cylinder of length $l$ with a cell and consider first the case without an oil excess. The extended mean energy density is then

$$
\begin{aligned}
& E=n w(c) \frac{2 \pi l}{c}+f\left(1-n \frac{2 \pi l}{c}\right) \\
& +p_{\mathrm{O}}\left[L_{\mathrm{O}}-n \pi l\left(\frac{1}{c}-\frac{h}{2}\right)^{2}\right]
\end{aligned}
$$

The constraints yield two equations for the determination of $c$ and $n l$.

$$
\left(1-\frac{h}{2} c\right)^{2}-2 L_{\mathrm{O}} c=0, \quad n l=\frac{c}{2 \pi}
$$

The vanishing of the derivatives with respect to $n$ and $c$ implies - note $d w / d c=\partial w / \partial c_{1}=-m_{1}$

$$
\begin{aligned}
& p_{\mathrm{O}}=2 c\left(1-\frac{h}{2} c\right)^{-2}(w-f) \\
& =c\left(1-\frac{h}{2} c\right)^{-1}\left(w-f+m_{1} c\right)
\end{aligned}
$$

and hence

$$
w-f=\frac{2-h c}{2+h c} m_{1} c
$$




$$
t_{1}=w-f+m_{1} c=\frac{4}{2+h c} m_{1} c
$$

and

$$
p_{\mathrm{O}}=\frac{8 m_{1} c^{2}}{(2-h c)(2+h c)}
$$

If an oil excess exists, then $p_{\mathrm{O}}=0$ leads to

$$
w-f=0, \quad m_{1}=0, \quad t_{1}=0
$$

So the cylinder is free of internal hoop forces and torques, and the curvature $c$ is now obtained from $-m_{1}=d w(c) / d c=0$.

\section{CONSTITUTIVE RELATIONS}

We define

$$
H=\frac{1}{2}\left(c_{1}+c_{2}\right), \quad D=\frac{1}{2}\left(c_{1}-c_{2}\right)
$$

and see that the local curvature tensor

$$
\mathbf{C}=H\left(\mathbf{a}_{1} \otimes \mathbf{a}_{1}+\mathbf{a}_{2} \otimes \mathbf{a}_{2}\right)+D\left(\mathbf{a}_{1} \otimes \mathbf{a}_{1}-\mathbf{a}_{2} \otimes \mathbf{a}_{2}\right)
$$

is the sum of an (isotropic) spherical part characterized by $H$ and an (anisotropic) saddle-splay part characterized by $D$. $H$ is an invariant of the curvature tensor and so is $D^{2}$, but $D$ is not, since it depends on the numbering of the axes. These invariants are related with the Gaussian curvature $K$ by

$$
K=H^{2}-D^{2}
$$

First, we restrict the energy density to a complete invariant quadratic expression in the curvatures and find two equivalent representations

$$
\begin{aligned}
& w(\mathbf{C})=\tilde{w}_{0}+\tilde{\lambda}_{1}\left(H-\tilde{H}_{0}\right)^{2}-\lambda_{2} K \\
& =w_{0}+\lambda_{1}\left(H-H_{0}\right)^{2}+\lambda_{2} D^{2}
\end{aligned}
$$

with the connections

$$
\begin{aligned}
& \tilde{\lambda}_{1}=\lambda_{1}+\lambda_{2}, \quad \tilde{\lambda}_{1} \tilde{H}_{0}=\lambda_{1} H_{0} \\
& \tilde{\lambda}_{1}\left(\tilde{w}_{0}-w_{0}\right)=\lambda_{1} \lambda_{2} H_{0}^{2}
\end{aligned}
$$

between the material constants. The common choice of the invariants is $(H, K)$, following Helfrich [13]. But the variables $\left(H, D^{2}\right)$ will be more useful in our later non-linear extension. The constant $w_{0}$ can henceforth be omitted since - as we already know - it does not influence the internal forces and torques of the film nor the search for the minimum conformation. A comparison with the behaviour of solid shells suggests the representation

$$
w=\lambda_{1}\left(\left(H-H_{0}\right)^{2}+\frac{1-v}{1+v} D^{2}\right)
$$

where $v$ is Poisson's ratio. The film with this constitutive behaviour enjoys its minimal energy density $w=0$ everywhere if it forms a sphere with the spontaneous curvature $H \equiv H_{0} \neq 0$ and radius $R=1 / H_{0}$ or a lamella with $H \equiv H_{0}=0$. A state of isotropic curvature is therefore preferred.

However, the experimental occurrence of bicontinuous surfaces shows that real isotropic films may instead prefer anisotropic curvatures. This phenomenon is an example of symmetry breaking, which was introduced in [9] and excludes a quadratic energy density. We will therefore extend the term containing $D^{2}$ in a non-linear manner. We follow [10] and choose

$$
w=\lambda_{1}\left(\left(H-H_{0}\right)^{2}+\frac{1-v}{1+v} \frac{\left(D^{2}-D_{0}^{2}\right)^{2}}{\Theta D_{0}^{2}+D^{2}}\right)
$$

The local minimal value $w=0$ of the energy density is now reached if $H=H_{0}$ and $D= \pm D_{0}$. (See Fig. (4).) This value can only be achieved everywhere in the structure in the two special cases $D_{0}=0$ (sphere or lamella) and $D_{0}= \pm H_{0}$ (cylinder). Otherwise, the free energy of any structure must be positive.

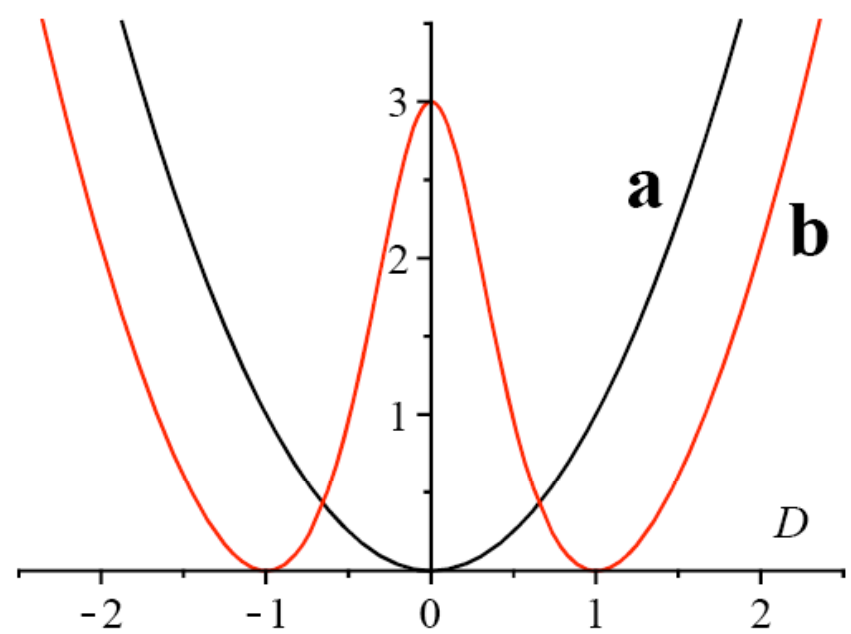

Fig. (4). The function $\left(D^{2}-D_{0}^{2}\right)^{2} /\left(\Theta D_{0}^{2}+D^{2}\right)$ from the saddlesplay part of the energy density according to equation (10) with $\Theta=1 / 3$. a: Case $D_{0}=0$, no symmetry breaking. b: Case $D_{0}=1$, symmetry breaking.

The choice (10) represents a model law, as complex as necessary and as simple as possible in order to admit symmetry breaking. The following computations are based either on this non-linear law or on Helfrich's law (9), which results from it by putting $D_{0}=0$. But we will also have to exploit the special case $v=1$, where (9) and (10) coincide and any surface with constant mean curvature $H \equiv H_{0}$ is a solution with zero energy. A broader study in [11] suggests a microscopic origin of symmetry breaking, but yields a more complex dependence of the energy density on the variables $H$ and $D^{2}$. 
A numerical examination reveals that the material parameter $\Theta$ has no essential influence on the qualitative behaviour of solutions. Therefore we always use the fixed value $\Theta=1 / 3$ in our computations.

Important remark: Our model law is not intended to fit experimental data of a special mixture. We only want to obtain qualitative insight, so that an excessive accuracy of the numerical results would be misleading.

\section{NUMERICAL COMPUTATION}

It is explained in appendix D that we endow the curved patch of our film within a cell with a mesh of gridpoints. Unless otherwise stated, all the following computations were performed with $16 \times 16$ gridpoints. Unknowns are the 256 values $\theta_{i j}$, which describe the shape of the placement of the film, and in addition the five quantities $n, f, p_{\mathrm{O}}, p_{\mathrm{w}}$, and $a$. (The length $a$ describes the magnitude of the placement and is explained below.) The extended energy density is thus represented as a function of 261 variables. The postulate that the first partial derivatives with respect to all of these variables vanish, yields 261 non-linear equations. These derivatives as well as the second derivatives required by the Newton-Raphson method are provided analytically.

It is obvious that this approach yields stationary values of the free energy but is not sufficient to guarantee a minimum. On the contrary, we will see that the non-linear equations may have more than one solution with rather different values of the energy.

If an oil excess exists, then the variable $p_{\mathrm{O}}$ is known to be zero, and the appertaining equation must be cancelled. The same is valid in the case of a water excess.

The variable $a$ may be identified with the characteristic length $e$ in case of tube3, tube4, tube6. But it is the fixed side length of the reference patch, if spheres or cylinders are to be treated numerically. (See appendix C.) In the latter case, $a$ must not be varied and the appertaining equation must be ignored. We learn in the next section that the same is true with tube 3 , tube 4 , tube 6 in the case $v=1$, where a family of solutions with parameter $e$ exists. So $a=e$ must be fixed to obtain a definite solution.

It is even possible to fix $n=1$ and $f=0$, to suppress the appertaining equations and to prescribe a constant energy density $w=w_{0}$. The numeric code is then adapted to solve problems from the theory of capillarity.

The quantity

$$
\delta=-\frac{6}{\pi} \int_{A_{\mathrm{C}}} K d A=\frac{6}{\pi} \int_{A_{\mathrm{C}}}\left(D^{2}-H^{2}\right) d A
$$

which we call the defect of the patch, is checked during each computation. If it differs markedly from the exact value, which is known a priori ( $c f$. Appendix C), then the value of the energy, which is obtained from a similar integral, will also be dubious.
The MAPLE code of the numeric scheme and input and output data of selected solutions can be found on the author's homepage [14].

\section{RESULTS}

The assignment of a characteristic length $e$ to a given structure is arbitrary. We obtain an expedient comparison of different structures by selecting the distance of two neighbouring vertices of the skeletal graph in case of tube3, tube4, tube6, and the diameter and twice the diameter - and hence $|e H|=2 \quad$ in case of sphere and cylinder, respectively. The concept of a characteristic length is not meaningful with lamellae.

The qualitative behaviour of solutions is strongly influenced by the material parameters $1-v, D_{0}$ and $H_{0}$, which may be either zero or positive. The six significant combinations are denoted by A, B, C, D, E, F and the corresponding properties of solutions compiled in Table $\mathbf{1}$. These findings are substantiated in the following sections.

\subsection{The Special Case $H_{0}=0$}

If we consider spheres (with $D \equiv 0$ ) then the energy density (10) reduces to

$$
w=\lambda_{1}\left(H^{2}+\frac{1-v}{1+v} \frac{D_{0}^{2}}{\Theta}\right)
$$

Its infimum is approached with $H \rightarrow 0$ and equals the energy density

$$
w=\lambda_{1} \frac{1-v}{1+v} \frac{D_{0}^{2}}{\Theta}
$$

of a lamella, but a minimum does not exist. So spheres cannot be encountered in cases A, B, E of Table 1 if no volume constraint exists.

The energy density of a cylinder (with $D^{2} \equiv H^{2}$ ) becomes

$$
\begin{aligned}
& w=\lambda_{1}\left(H^{2}+\frac{1-v}{1+v} \frac{\left(H^{2}-D_{0}^{2}\right)^{2}}{\Theta D_{0}^{2}+H^{2}}\right) \\
& =\lambda_{1}\left(\tilde{H}^{2}+\frac{1-v}{1+v} \frac{\left(\tilde{H}^{2}-D_{0}^{2}\right)^{2}}{\Theta D_{0}^{2}+\tilde{H}^{2}}+\frac{2}{1+v} \frac{\left(H^{2}-\tilde{H}^{2}\right)^{2}}{\Theta D_{0}^{2}+H^{2}}\right)
\end{aligned}
$$

and attains a minimum at

$$
|H|=\tilde{H} \equiv D_{0} \sqrt{(1+\Theta) \sqrt{\frac{1-v}{2}}-\Theta}
$$

provided that this value is real and positive, which requires $D_{0}>0$ and

$$
\sqrt{\frac{1-v}{2}}>\frac{\theta}{1+\theta}
$$


Table 1. Influence of the Material Parameters on the Solution Properties

\begin{tabular}{|c|c|c|c|c|c|c|}
\hline Case & $\mathbf{A}$ & B & $\mathbf{C}$ & D & $\mathbf{E}$ & $\mathbf{F}$ \\
\hline \multicolumn{7}{|c|}{ Material parameters } \\
\hline$D_{0}$ & 0 & $>0$ & $>0$ & 0 & - & - \\
\hline$\omega$ & - & 0 & $>0,<1$ & 1 & - & - \\
\hline$c_{\mathrm{M}}$ & 0 & $>0$ & $>0$ & $>0$ & 0 & $>0$ \\
\hline SB & $\mathrm{N}$ & $\mathrm{Y}$ & $\mathrm{Y}$ & $\mathrm{N}$ & $\mathrm{N}$ & $\mathrm{N}$ \\
\hline \multicolumn{7}{|c|}{ Properties in case of no volume constraint } \\
\hline $\mathrm{M}-\mathrm{C}$ & $\mathrm{N}$ & $\mathrm{Y}, \mathrm{N}$ & $\mathrm{Y}$ & $\mathrm{Y}$ & $\mathrm{N}$ & $\mathrm{Y}$ \\
\hline M-T3 & $\mathrm{N}$ & $(\mathrm{Y})$ & $(\mathrm{Y})$ & $\mathrm{Y}$ & $\mathrm{N}$ & $\mathrm{Y}$ \\
\hline M-T4,T6 & $\mathrm{N}$ & $(\mathrm{Y})$ & $(\mathrm{Y})$ & $\mathrm{Y}$ & $\mathrm{D}, \mathrm{P}$ & $\mathrm{Y}$ \\
\hline \multicolumn{7}{|c|}{ Properties in case of an oil volume constraint } \\
\hline $\mathrm{AD}-\mathrm{S}, \mathrm{C}$ & $\mathrm{Y}$ & $\mathrm{Y}$ & $\mathrm{Y}$ & $\mathrm{Y}$ & $\mathrm{Y}$ & $\mathrm{Y}$ \\
\hline AD-T3 & - & $\mathrm{N}$ & $\mathrm{N}$ & $\mathrm{N}$ & - & $\mathrm{N}$ \\
\hline AD-T4,T6 & $\mathrm{Y}$ & A & $\mathrm{N}$ & $\mathrm{N}$ & $\mathrm{D}, \mathrm{P}$ & $\mathrm{N}$ \\
\hline CMC-S,C & $\mathrm{Y}$ & $\mathrm{Y}$ & $\mathrm{Y}$ & $\mathrm{Y}$ & $\mathrm{Y}$ & $\mathrm{Y}$ \\
\hline
\end{tabular}

Abbreviations: SB: Symmetry breaking. Y: Yes. N: No. A: Approximately. D, P: Yes, with a minimal surface ( $H \equiv 0)$ of Schwarz (D with tube4 and P with tube6). ZE: Existence of a solution with zero energy $(w \equiv 0)$. M: Existence of a solution with minimal energy. AD: Affine diminution of the solution surface with decreasing oil length. CMC: The solution is a surface with constant mean curvature. L, S, C, T3, T4, T6: Solution within the families lamella, sphere, cylinder, tube3, tube 4 , and tube6.

The brackets around $\mathrm{Y}$ indicate that these statements were found with the parameters $\theta=1 / 3$ and $v=0.9$ but need not hold generally. The alternative $\mathrm{Y}$, $\mathrm{N}$ with M-C refers to the fulfilment of the condition (12).

Otherwise, an infimum is approached with $H \rightarrow 0$, but a minimum does not exist. This is especially the case in the absence of symmetry breaking $\left(D_{0}=0\right.$ or $\left.v=1\right)$ and therefore cylinders cannot be encountered in cases A and $\mathrm{E}$ of Table 1 without a volume constraint.

If an oil constraint exists, then spheres and cylinders are possible solutions, but their energy is positive in any case since $H>0$.

Next, we consider the tube families. If $v=1$ (case E), then the energy density $w=\lambda_{1} H^{2}$ attains the minimum value $w \equiv 0$ if $H \equiv 0$. This is valid with the minimal surfaces $\mathrm{D}$ and $\mathrm{P}$ in case of tube 4 and tube6, respectively, while no such minimal surface is contained in the family tube 3 . Since the characteristic length $e$ of the surfaces D and P is arbitrary, they may enclose any amount of oil but also allow for an oil excess. So the notion of a volume constraint dissolves in this case.

If $v<1$ and $D_{0}=0$ (case A) then the energy density becomes

$$
w=\lambda_{1}\left(H^{2}+\frac{1-v}{1+v} D^{2}\right)
$$

Now, if there is no volume constraint, let us consider an equilibrium solution within tube4 or tube6 with fixed characteristic length $e$. (It will be rather close to a D or $\mathrm{P}$ 
surface. No such solution exists in case of tube3.) If we apply an affine magnification with factor $\chi$ then $e \rightarrow \chi e$ and $w \rightarrow w / \chi^{2}$. So the mean energy density approaches its infimum $\bar{w}=0$ if $\chi \rightarrow \infty$, but a minimum does not exist within these families. On the other hand, if $v<1$ and $D_{0}>0$ (case B, with symmetry breaking), then our numerical computations produce solutions with minimal energy in each of the families tube3, tube4, tube6.

Next, we apply a volume constraint and let the amount of oil decrease. Then the tube 4 and tube6 solutions undergo an exact (case A) or approximate (case B) affine diminution. However, not the surfaces D or P do minimize the energy. They cancel the first term of the energy density, which depends on $H$, but let the second term, which depends on $D$, remain too big. The resulting surface, instead, has not a constant mean curvature, and its energy is positive. These findings remain true in case $\mathrm{B}$, where even a tube 3 solution exists. But the latter does by no means undergo an affine diminution if the amount of oil decreases.

\subsection{The Special Case $H_{0} \neq 0$ and $v=1$}

This is case $\mathrm{F}$ of Table $\mathbf{1}$. The energy density reduces to

$$
w=\lambda_{1}\left(H-H_{0}\right)^{2}
$$

It does not comprise the material constant $D_{0}$ and hence shows no symmetry breaking. Any surface with constant mean curvature $H \equiv H_{0}$ possesses the minimum possible energy density $w \equiv 0$. If no volume constraint exists, then spheres and cylinders with the fixed value $\left|e H_{0}\right|=2$ are admissible, while solutions for tube3, tube4, tube6 exist with various values of $e H_{0}$. The maxima of their absolute values which could be achieved numerically were $2.45,2.30$, and 2.08 , respectively. Lamellae are of no interest since they require the positive energy density $w=\lambda_{1} H_{0}^{2}>0$.

In case of an oil volume constraint, we will have $H \neq H_{0}$ and $w \neq 0$ with spheres and cylinders. On the other hand, tubes may exhibit solutions with $H \equiv H_{0}$ and $w \equiv 0$, but various values of $e$. They are, of course, not affine and may enclose different amounts of oil. Here again, the notion of a volume constraint becomes vague.

\subsection{No Volume Constraints}

This implies the existence of both an oil and a water excess, when we handle tube 4 and tube6. In case of sphere, cylinder, and tube 3 , the water outside of these structures is part of the microemulsion and a water excess cannot be observed, while oil is not only contained within the structures but also present as an excess phase.

We define two constitutive parameters, the dimensionless value

$$
\omega=\frac{2}{\pi} \arctan \frac{H_{0}}{D_{0}}
$$

and the material curvature

$$
c_{\mathrm{M}}=\sqrt{H_{0}^{2}+\frac{1-v}{1+v} \frac{1}{\Theta} D_{0}^{2}}
$$

The oil length of a cell $l_{\mathrm{O}}=V_{\mathrm{OC}} / A_{\mathrm{C}}$ does not only depend on the placement of the middle surface but also on the thickness $h$ of the film. We restrict the following presentation to the case $h \rightarrow 0$.

The Figs. (5-8) cover the range from $\omega=0$ to $\omega=1$ (case B, C, D of Table 1) with the choice $v=0.9$, and some of them also give the continuation into negative values of $\omega$ and hence of $H_{0}$. The Figs. (5,6 and 7) present the dimensionless quantities $l_{\mathrm{O}} c_{\mathrm{M}}, e H_{0}$, and $e / l_{\mathrm{O}}$ as functions of $\omega$. It is noteworthy that these quantities are not determined in any way by constitutive parameters with tube 3 , tube 4 , tube 6 if $v=1$ (case $\mathrm{E}$ and $\mathrm{F}$ with tube6 and tube4, case F only with tube3). The dimensionless energy $\bar{w} /\left(\lambda_{1} c_{M}^{2}\right)$, given in Fig. (8) as a function of $\omega$, is generally not zero in contrast to the case $v=1$, while its value is equal to 1 - independent of $v$ - in case of lamellae. If we fix the constitutive parameter $\omega$, then we infer from the figure which structure possesses the minimal energy. These are consecutively tube6, tube4, tube 3 , cylinder, and sphere, if increasing values of $\omega$ from 0 to 1 are considered. Below $\omega \approx 0.3$, we encounter a threephase system (microemulsion and oil and water excesses) and above a two-phase system (microemulsion and oil excess). The tuning of $\omega$ can experimentally be achieved, e.g., by a change of temperature, which influences the preferred mean curvature $H_{0}$.

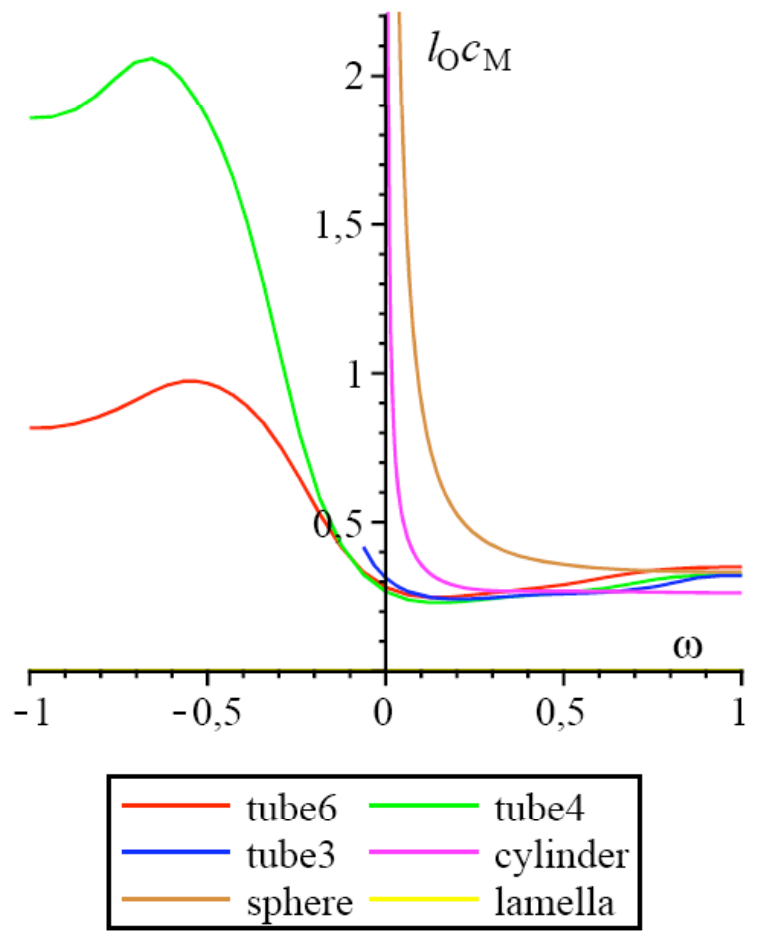

Fig. (5). No volume constraint. Dependence of $l_{\mathrm{O}} c_{\mathrm{M}}$ on $\omega$ in the case $v=0.9$. Compares the oil content of different structures. 


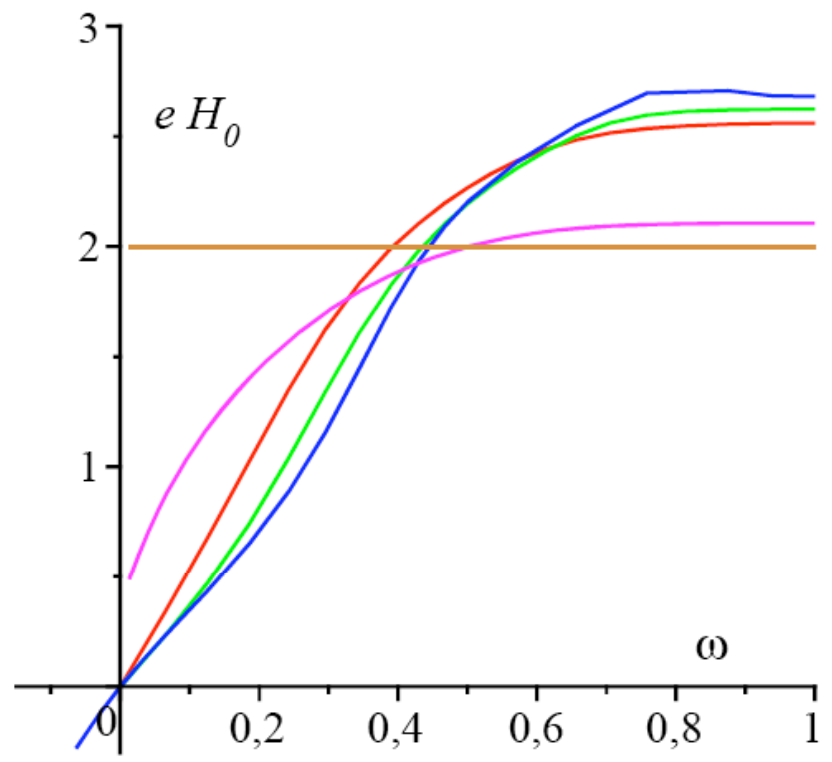

Fig. (6). No volume constraint. Dependence of $e H_{0}$ on $\omega$ in the case $v=0.9$. Indicates the magnitude of the structures.

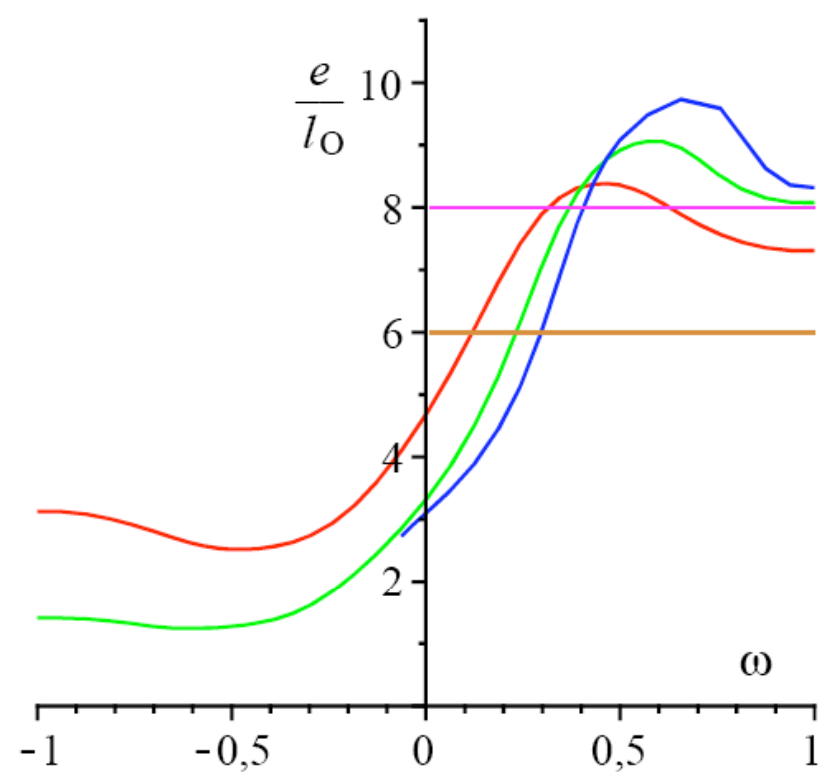

Fig. (7). No volume constraint. Dependence of $e / l_{\mathrm{O}}$ on $\omega$ in the case $v=0.9$. Characterizes the shape of the structures.

Figs. (9a, 9b) show $e / l_{0}$ as a function of $l_{0} H_{0}$ with $v=0.9$ (case B, C, D) and $v=1$ (case E, F), respectively. Remarkable differences appear with cylinder and tube3. The diagrams are truncated on the left-hand side.

Fig. (10) gives the mean value and the standard deviation of $H / H_{0}$ as functions of $e H_{0}$ in the case $v=0.9$ and compares it with the value $H / H_{0} \equiv 1$ of the case $v=1$.

The mean value and the standard deviation of $e D$ as functions of $e H_{0}$ with $v=0.9$ and $v=1$ are represented in Figs. (11a, 11b), respectively. We also include the diagram of $e D_{0}$ in the case $v=0.9$, while $D_{0}$ is not defined if $v=1$. The variable $e H_{0}$ of Figs. $(\mathbf{1 0}, \mathbf{1 1})$ depends on $\omega$ according to Fig. (6) if $v=0.9$, but may be any value within some range mentioned in the previous section if $v=1$.

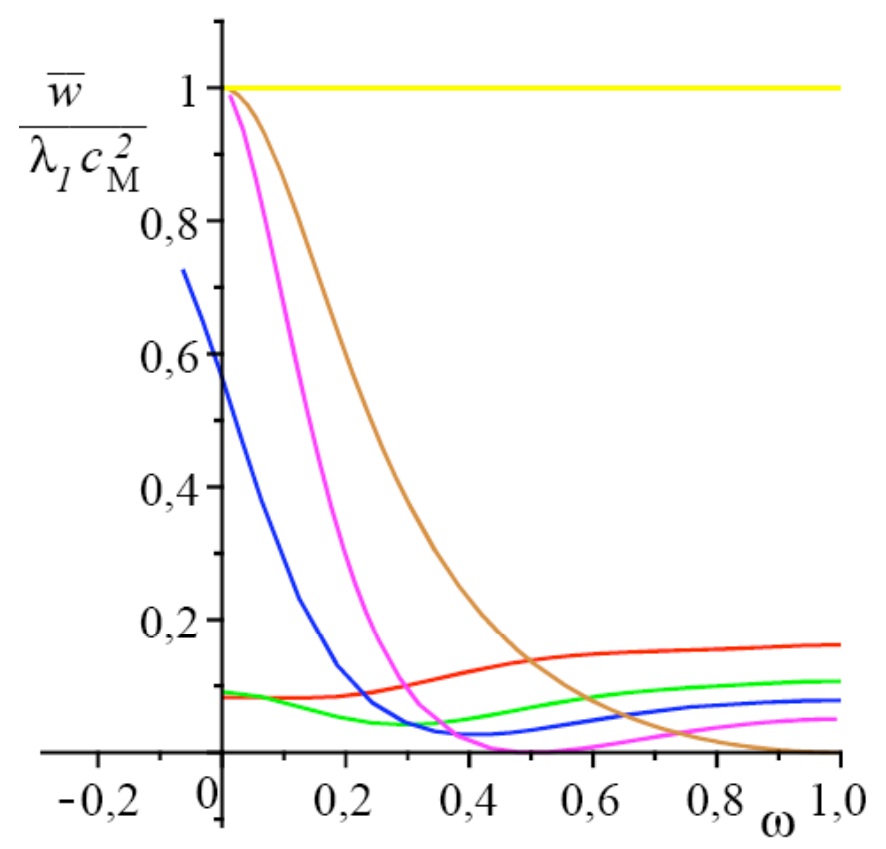

Fig. (8). No volume constraint. Dependence of $\bar{w} /\left(\lambda_{1} c_{M}^{2}\right)$ on $\omega$ in the case $v=0.9$. Compares the energy of different structures. The yellow line gives the constant value 1 of a lamella.

The Figs. (5-11) remain valid if we substitute $H_{0} \rightarrow-H_{0}, \quad \omega \rightarrow-\omega, \quad l_{\mathrm{O}} \rightarrow l_{\mathrm{w}}$ and assume that the spheres, cylinders, and tube 3 surfaces enclose water instead of oil.

Figs. (12a, 12b, 12c) show placements of the elementary patches of tube6, tube4, and tube3, computed with $v=0.9, H_{0}=0, D_{0}>0$ (case B). The tube6 and tube4 solutions are adjacent to the minimal surfaces $\mathrm{D}$ and $\mathrm{P}$, which would result in case $v=1$, while a tube 3 solution does not exist in that case.

\subsection{Oil Volume Constraint}

Remember the constraints

$$
A=N A_{\mathrm{C}}, \quad V_{\mathrm{O}} \geq N V_{\mathrm{OC}}
$$

which imply

$$
L_{\mathrm{O}} \equiv \frac{V_{\mathrm{O}}}{A} \geq \frac{V_{\mathrm{OC}}}{A_{\mathrm{C}}} \equiv l_{\mathrm{O}}
$$

If an oil excess exists then the oil length $L_{O}$ of the mixture is greater than the oil length $l_{\mathrm{O}}$ of the structure. Otherwise, the two lengths are equal. Now consider Figs. (13-17). The variable on the horizontal axis is always $L_{\mathrm{O}} c_{\mathrm{M}}$. 


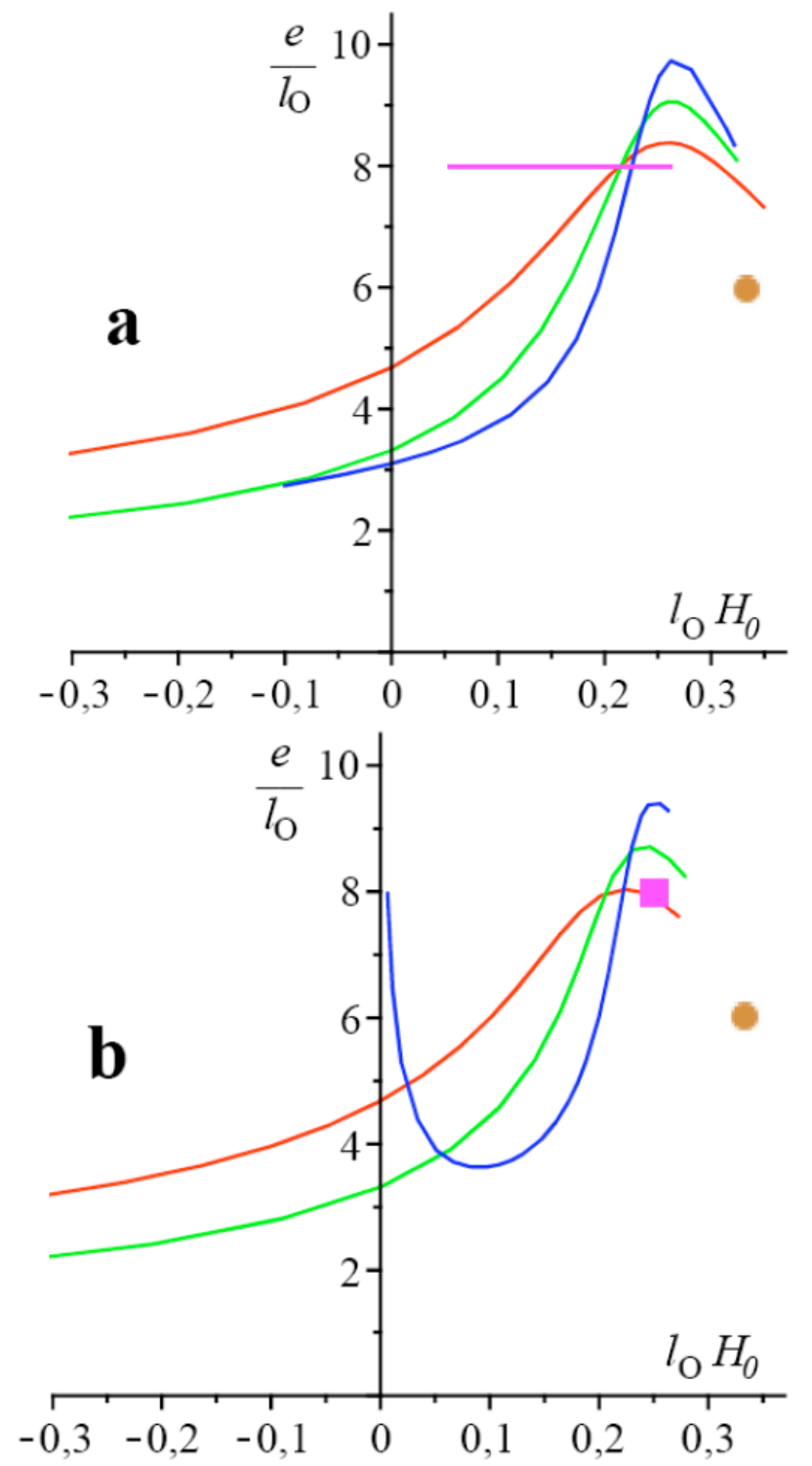

Fig. (9). No volume constraint. Dependence of $e / l_{\mathrm{O}}$ on $l_{\mathrm{O}} H_{0}$. a: Case $v=0.9$. b: Case $v=1$.

Let us regard a sufficiently small positive value of this variable. If $v<1$ then there will be no structure within any of our families that allows an oil excess, and so we have $l_{\mathrm{O}} c_{\mathrm{M}}=L_{\mathrm{O}} c_{\mathrm{M}}$. If we increase the amount of oil and hence $L_{\mathrm{O}} c_{\mathrm{M}}$, we will reach a critical value, which can be read off from Fig. (5), such that the structure can exist without the volume constraint, and with zero oil pressure. That limit is marked by a big dot on the curves. If $L_{\mathrm{O}} c_{\mathrm{M}}$ is further increased, then the structure and all its properties, e.g. $l_{\mathrm{O}} c_{\mathrm{M}}$, remain constant while the surplus of oil forms an excess phase. On the other hand, if $v=1$, then the big dot indicates the maximum possible value of $l_{\mathrm{O}} c_{\mathrm{M}}$. If $L_{\mathrm{O}} c_{\mathrm{M}}$ exceeds this value then an oil excess inevitably exists. But we cannot exclude the possibility that such an excess exists with smaller values of $L_{\mathrm{O}} c_{\mathrm{M}}$, since the energy of the film has always its minimum possible value zero.

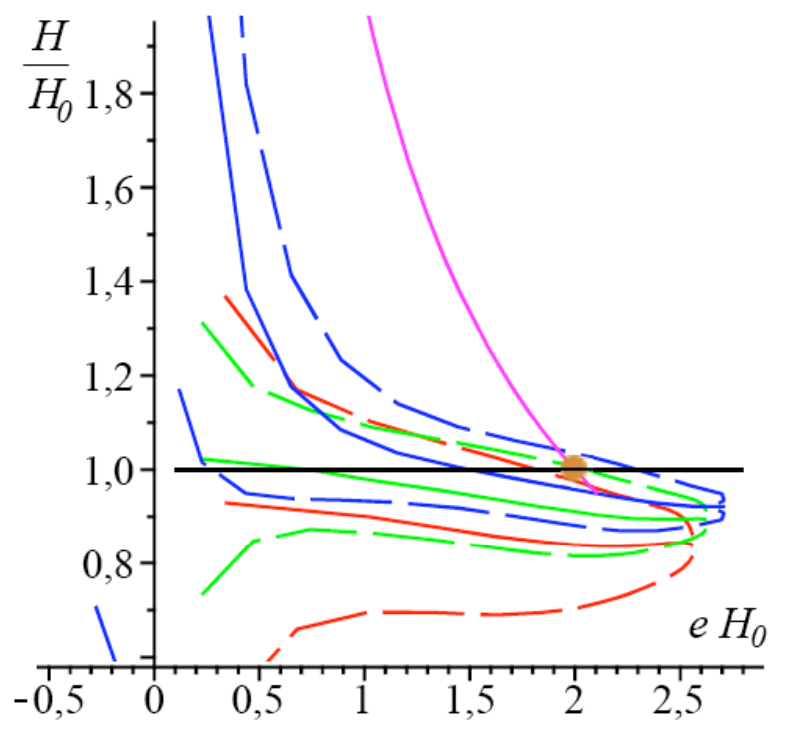

Fig. (10). No volume constraint. Dependence of $H / H_{0}$ on $e H_{0}$ in the case $v=0.9$. Solid line: Mean value. Dashed line: Mean value \pm standard deviation. The black line gives the constant value 1 of the case $v=1$.

The diagrams $\mathbf{A B}, \mathbf{C}, \mathbf{D}$ of each figure are computed with $v=0.9$. Moreover, the diagram $\mathbf{A B}$ is based on $H_{0}=0$; the symbols represent $D_{0}=0$ and hence $c_{\mathrm{M}}=0$ and $L_{\mathrm{O}} c_{\mathrm{M}}=0$ with any $L_{\mathrm{O}}$ (case A of Table $\mathbf{1}$ ), the curves represent $D_{0}>0$ and hence $\omega=0$ and $L_{\mathrm{O}} c_{\mathrm{M}}>0$ (case B). Diagram C is based on $H_{0} / D_{0}=0.7$ or $\omega=0.388$ (case C) and diagram $\mathbf{D}$ on $H_{0}>0, D_{0}=0$ or $\omega=1$ (case D). Diagram EF is computed with $v=1$; the symbols represent $H_{0}=0$ and hence $c_{\mathrm{M}}=0$ and $L_{\mathrm{O}} c_{\mathrm{M}}=0$ with any $L_{\mathrm{O}}$ (case $\mathrm{E}$ of Table 1), the curves represent $H_{0}>0$ and hence $L_{\mathrm{O}} c_{\mathrm{M}}>0$ (case F).

Particularly, Fig. (13) gives the ratio $e / l_{\mathrm{O}}$ and Fig. (14) presents the mean value and the standard deviation of $e D$ of the three tube structures. Note that Fig. (13F) is identical with Fig. (9b) as far as the tube structures are concerned.

If a reduction of the amount of oil and hence of $L_{\mathrm{O}}$ induces an affine diminution of the structure, then the values of $e / l_{\mathrm{O}}$ and $e D$ remain constant. This is exactly the case with spheres and cylinders. Moreover, if $H_{0}=0$, then it is realized with tube 6 and tube 4 exactly if $v<1, D_{0}=0$ (case A) or $v=1$ (case E) and approximately if $v<1, D_{0}>0$ (case B). On the other hand, if $H_{0}>0$, then the values of both $e / l_{\mathrm{O}}$ and $e D$ of the tube structures vary markedly and the characteristic length $e$, although decreasing monotonically, is by no means proportional to the oil length $l_{\mathrm{O}}$. 

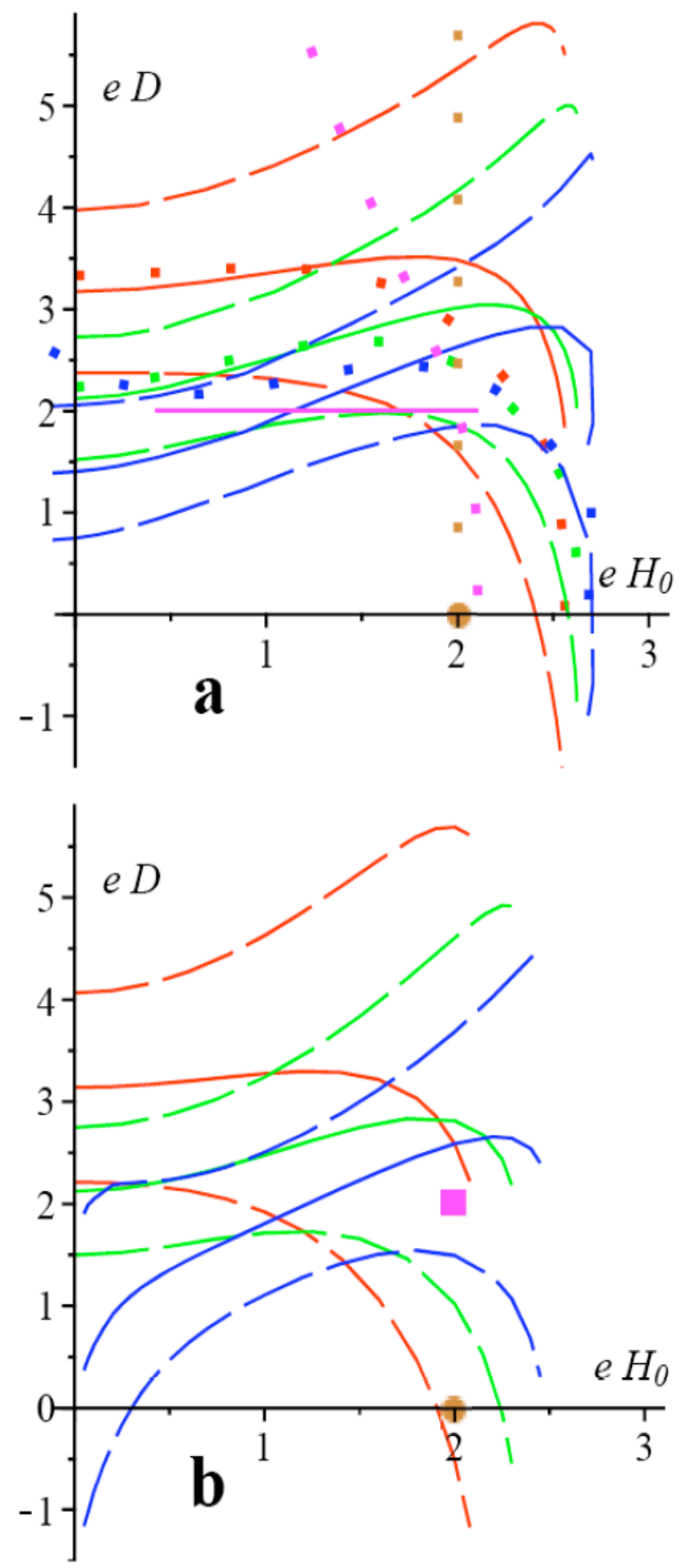

Fig. (11). No volume constraint. Dependence of $e D$ on $e H_{0}$. Solid line: Mean value. Dashed line: Mean value \pm standard deviation. a: Case $v=0.9$. The dotted line gives $e D_{0}$. b: Case $v=1$.

Fig. (15) presents the mean value and the standard deviation of $e\left(H-H_{0}\right)$ of the three tube structures and the values of cylinders and spheres.
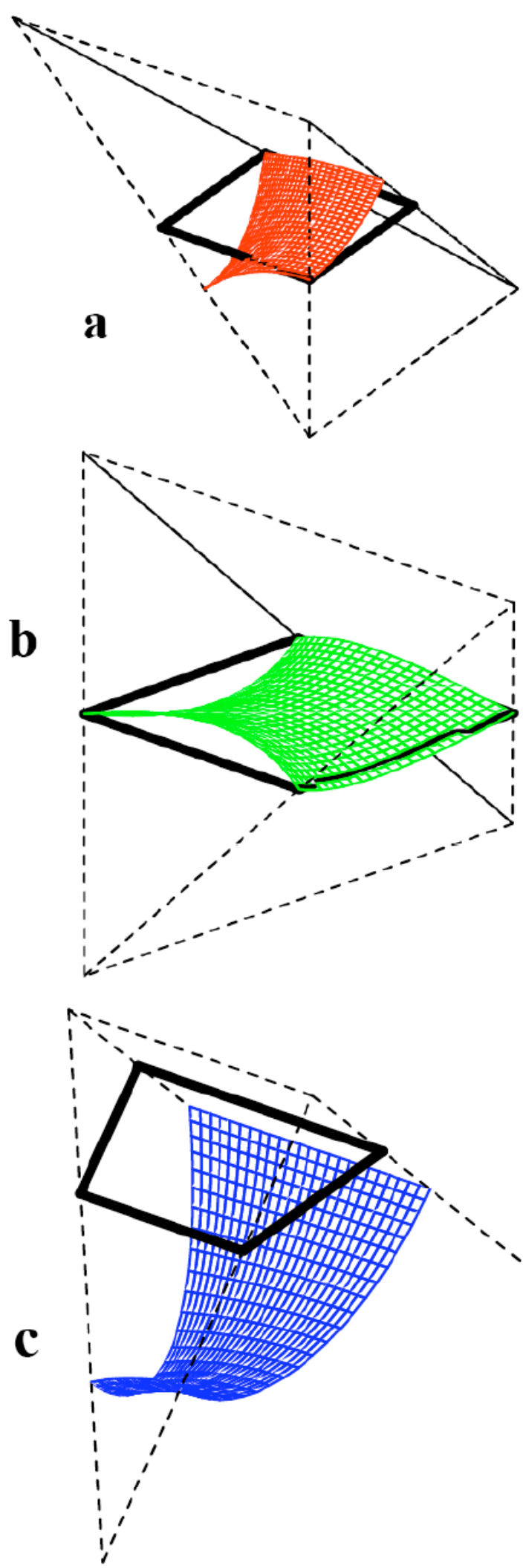

Fig. (12). No volume constraint. Placement of the elementary patch in the case $v=0.9, H_{0}=0, D_{0}>0$. The boundary of the quadrilateral plane reference patch is marked by a thick line, the thin lines indicate the intersection of two planes of symmetry and delimit the cell volume. a: tube6. b: tube4. c: tube3, enclosing either oil or water. 

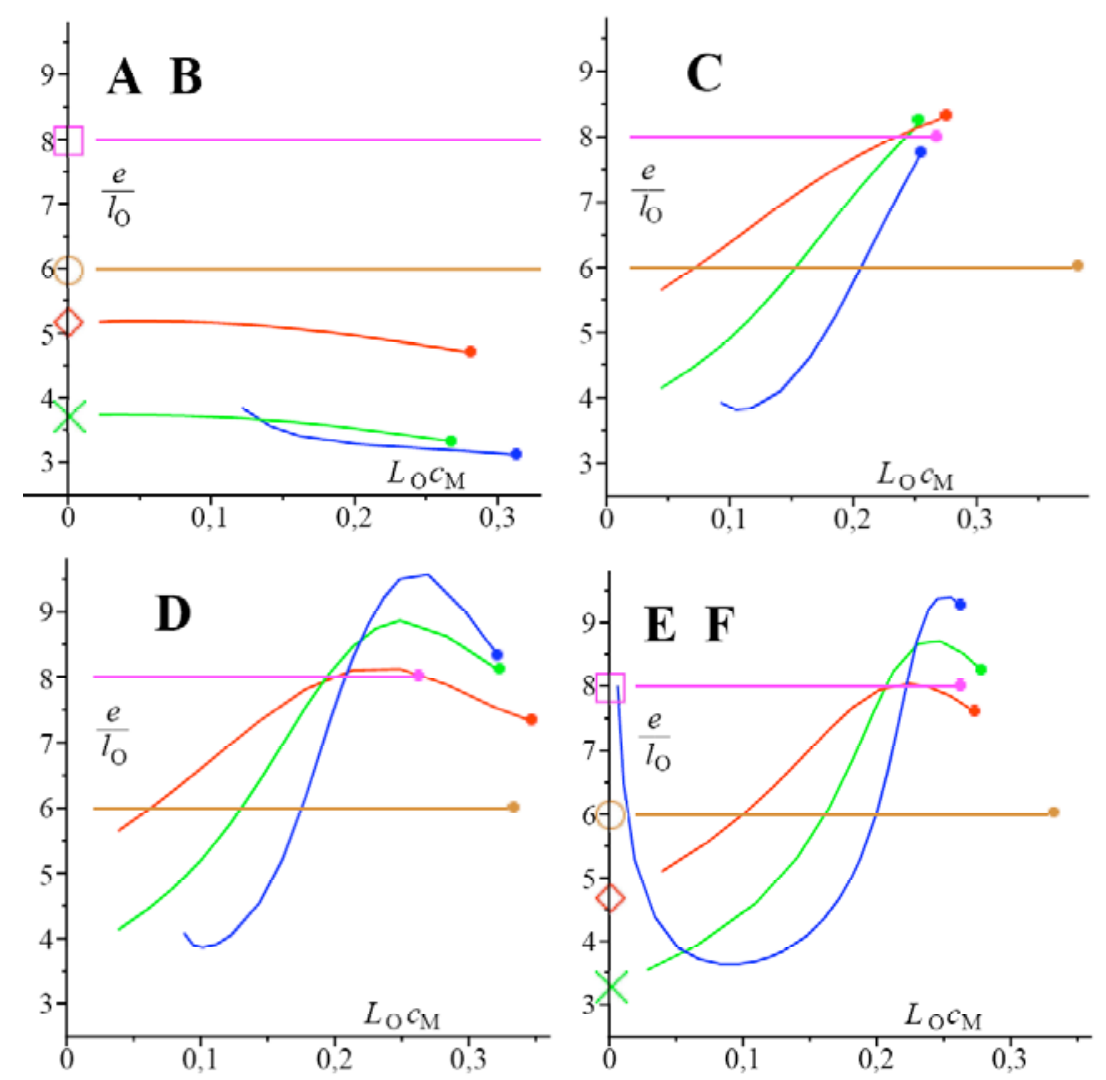

Fig. (13). Oil volume constraint. Dependence of $e / l_{\mathrm{O}}$ on $L_{\mathrm{O}} c_{\mathrm{M}} \cdot \mathbf{A}, \mathbf{B}, \mathbf{C}, \mathbf{D}, \mathbf{E}, \mathbf{F}$ : Cases according to Table 1.
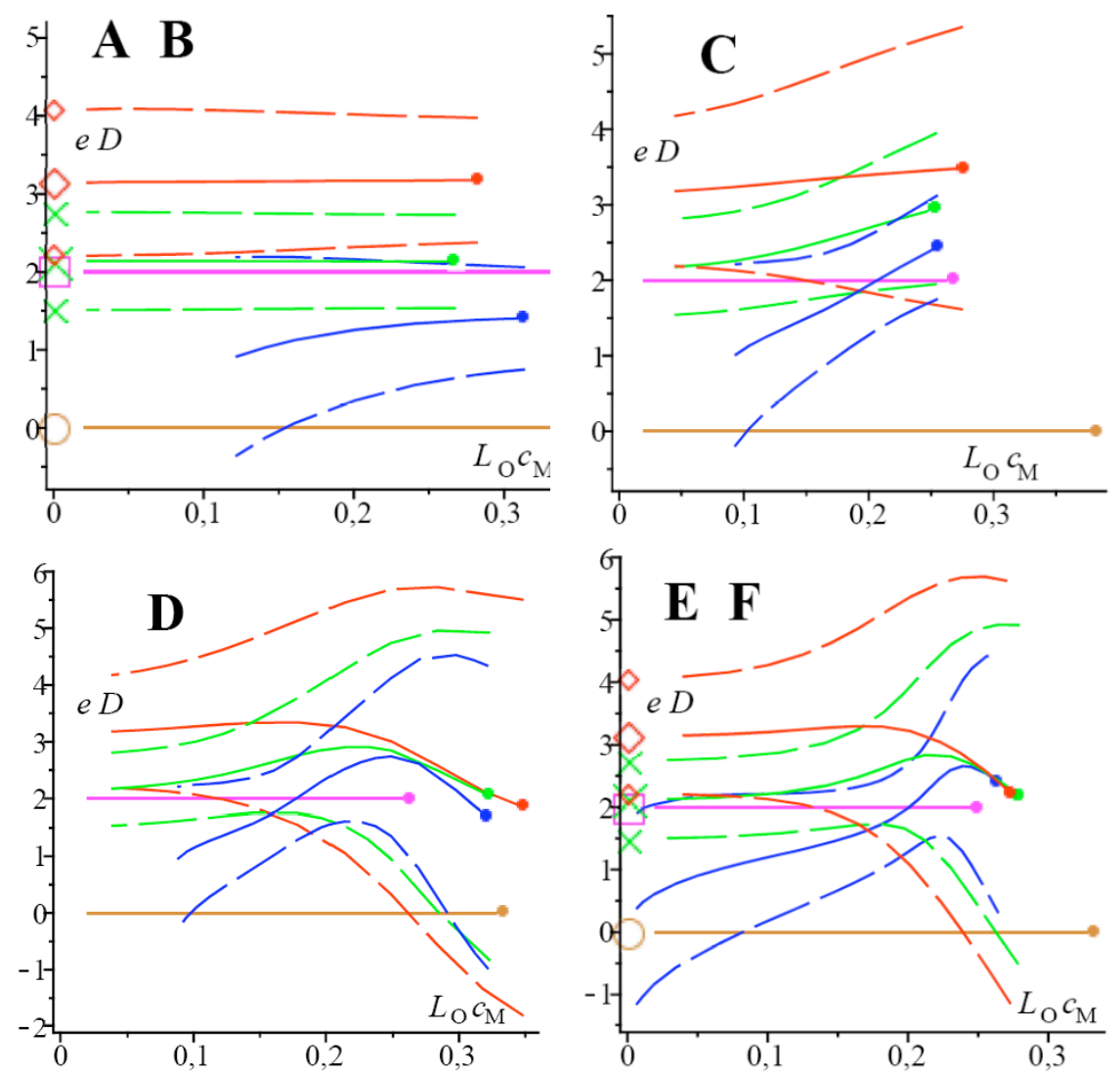

Fig. (14). Oil volume constraint. Dependence of $e D$ on $L_{\mathrm{O}} c_{\mathrm{M}}$. Solid line: Mean value. Dashed line: Mean value \pm standard deviation. 

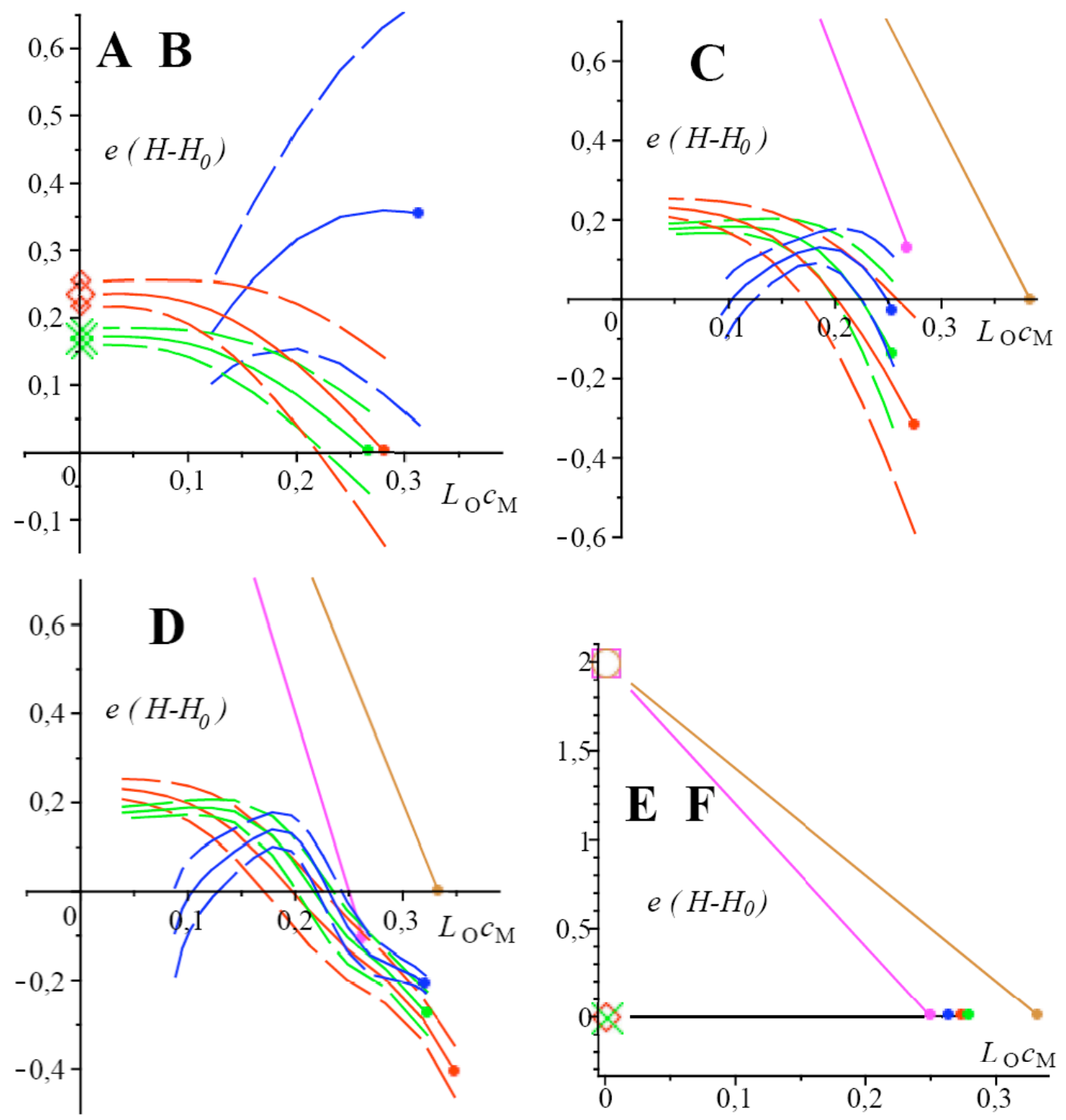

Fig. (15). Oil volume constraint. Dependence of $e\left(H-H_{0}\right)$ on $L_{\mathrm{O}} c_{\mathrm{M}}$. Solid line: Mean value. Dashed line: Mean value \pm standard deviation. The constant value 2 of spheres and cylinders in case $\mathbf{A}$ and $\mathbf{B}$ lies outside the displayed range. The constant value 0 of tube6, tube4, tube 3 in case $\mathbf{F}$ is represented by a black line.

Fig. (16) gives the dimensionless energy $\bar{w} L_{\mathrm{O}}^{2} / \lambda_{1}$. On the right-hand side of the phase transition (characterized by the big dot on the curve), the mean energy density $\bar{w}$ remains constant and the (dashed) curves are therefore parabolic. If decreasing values of $L_{O}$ are considered, then the structures with minimal energy are seen to be, consecutively, tube6, tube 4 , and lamella in case B $(\omega=0)$, cylinder, tube 3 , and lamella in case $\mathrm{C}(\omega=0.388)$ and sphere, cylinder, tube 3 , and lamella in case D $(\omega=1)$. A water excess is present with tube 6 and tube 4 , but not with the other structures.

Fig. (17) presents the dimensionless oil pressure $p_{\mathrm{O}} L_{\mathrm{O}}^{3} / \lambda_{1}$ (negative, as we know). If $v=1$ then the tubes have zero energy and the oil pressure is zero, too (case $\mathrm{E}$ and F with tube6 and tube4, case F only with tube3).

\subsection{Oil and Water Volume Constraint}

We discuss an example: Tube 4 , case B $(v=0.9$, $H_{0}=0, D_{0}>0$ ), $L_{\mathrm{w}} / L_{\mathrm{O}}=1.15$ (Such a constant ratio is maintained if the amount of amphiphile is raised.). The green curves in Figs. (18a, 18b, 18c) give $e / L_{\mathrm{O}}, e\left(H-H_{0}\right) \equiv e H$, and $p_{\mathrm{O}} L_{\mathrm{O}}^{3} / \lambda_{1}$ together with $p_{\mathrm{w}} L_{\mathrm{O}}^{3} / \lambda_{1}$, respectively, as functions of $L_{\mathrm{O}} c_{\mathrm{M}}$. The black curves - taken from Figs. (13B, 15B and 17B) for the sake of comparison - describe the behaviour if only the oil volume constraint is present. The fact that now $e / L_{\mathrm{O}}$ and $e \bar{H}$ remain constant suggests an affine diminution. However, the standard deviation of $e H$ diminishes with decreasing amount of oil and water, so that the tube tends towards a surface with constant mean curvature. Since $L_{\mathrm{w}} / L_{\mathrm{O}} \neq 1$, this mean curvature differs 

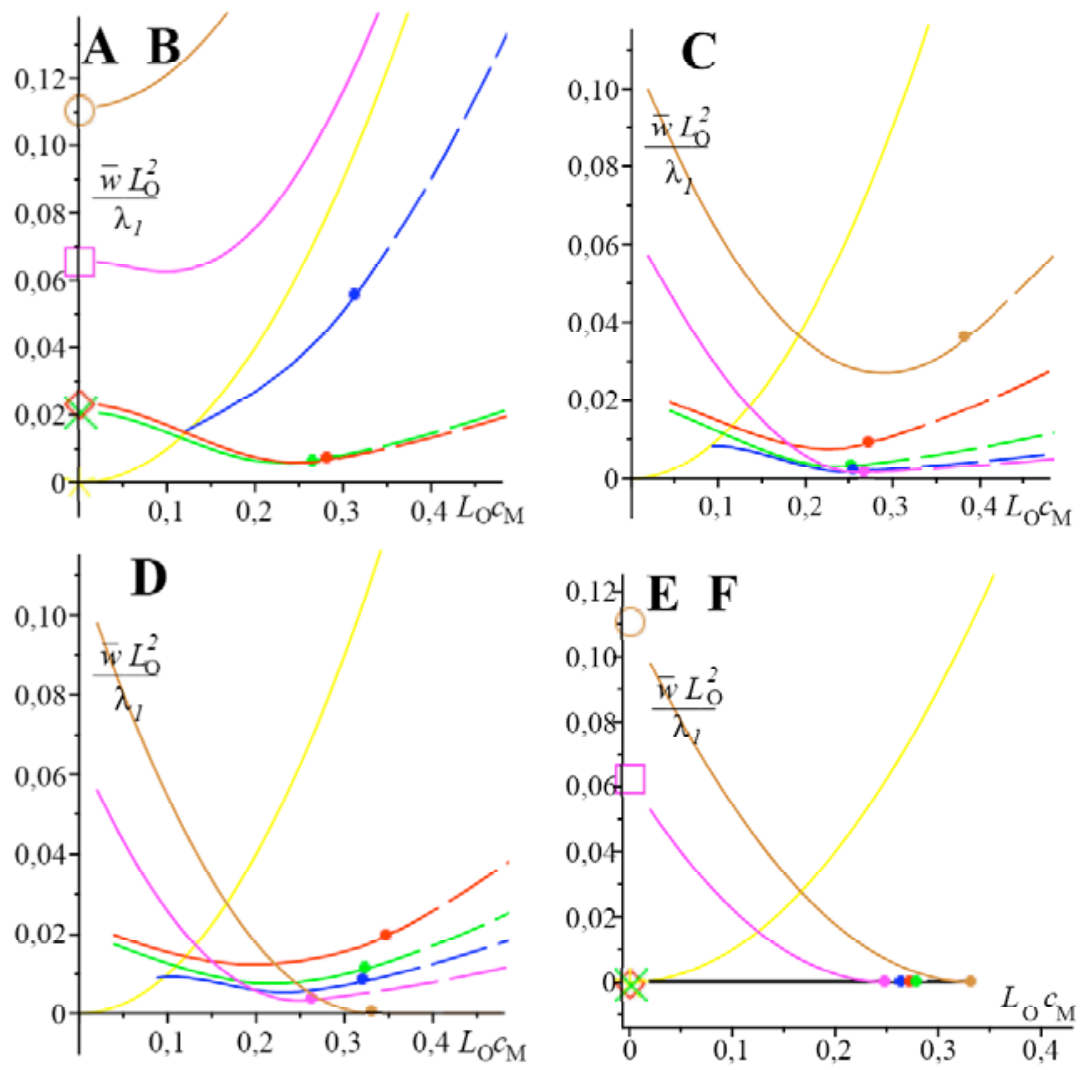

Fig. (16). Oil volume constraint. Dependence of $\bar{w} L_{\mathrm{O}}^{2} / \lambda_{\mathrm{I}}$ on $L_{\mathrm{O}} c_{\mathrm{M}}$. The constant value 0 of tube6, tube4, tube3 in case $\mathbf{F}$ is represented by a black line.
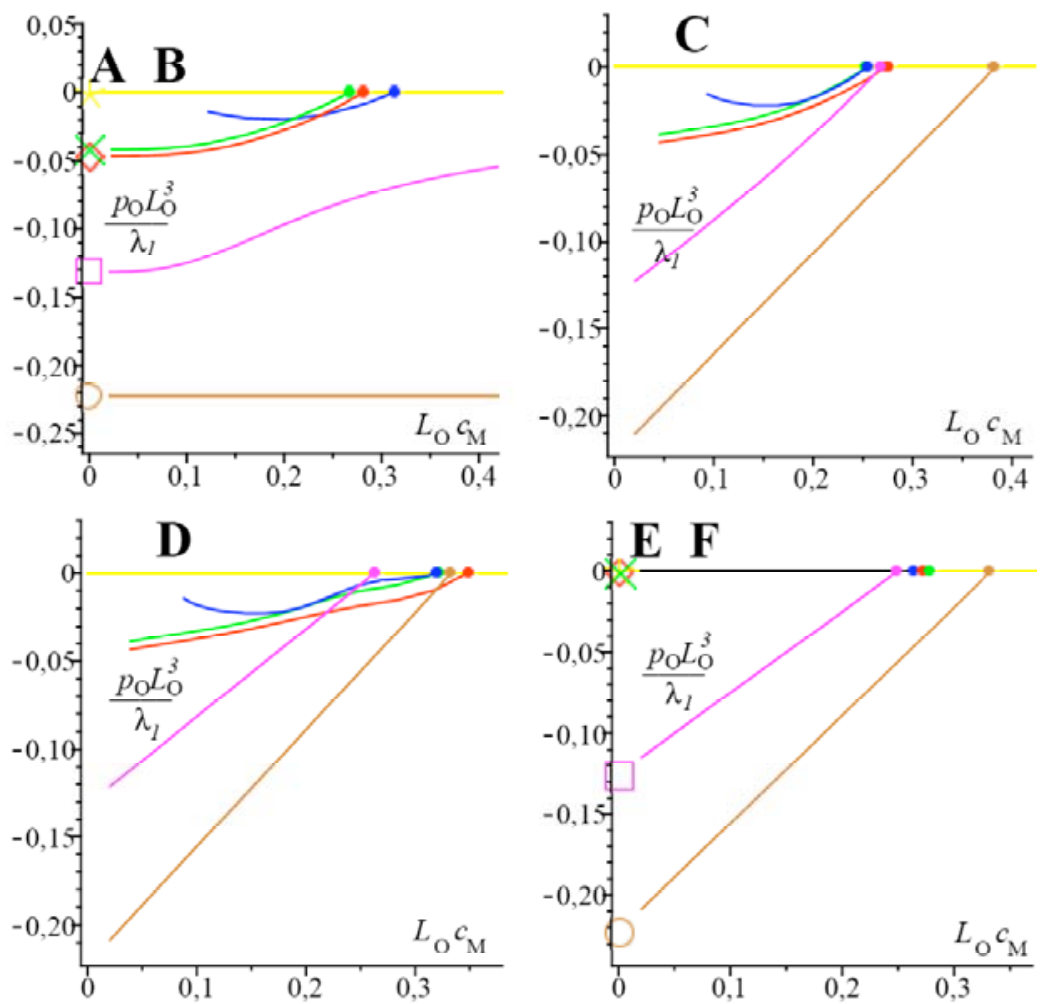

Fig. (17). Oil volume constraint. Dependence of $p_{\mathrm{O}} L_{\mathrm{O}}^{3} / \lambda_{1}$ on $L_{\mathrm{O}} c_{\mathrm{M}}$. The constant value 0 of tube6, tube4, tube3 in case $\mathbf{F}$ is represented by a black line. 
from $H_{0}=0$ and increases nearly proportional to $1 / L_{\mathrm{O}}$. We see that the absolute value of the oil pressure can now be smaller, because there is also a (negative) water pressure due to the water volume constraint, which helps to contract the tube.
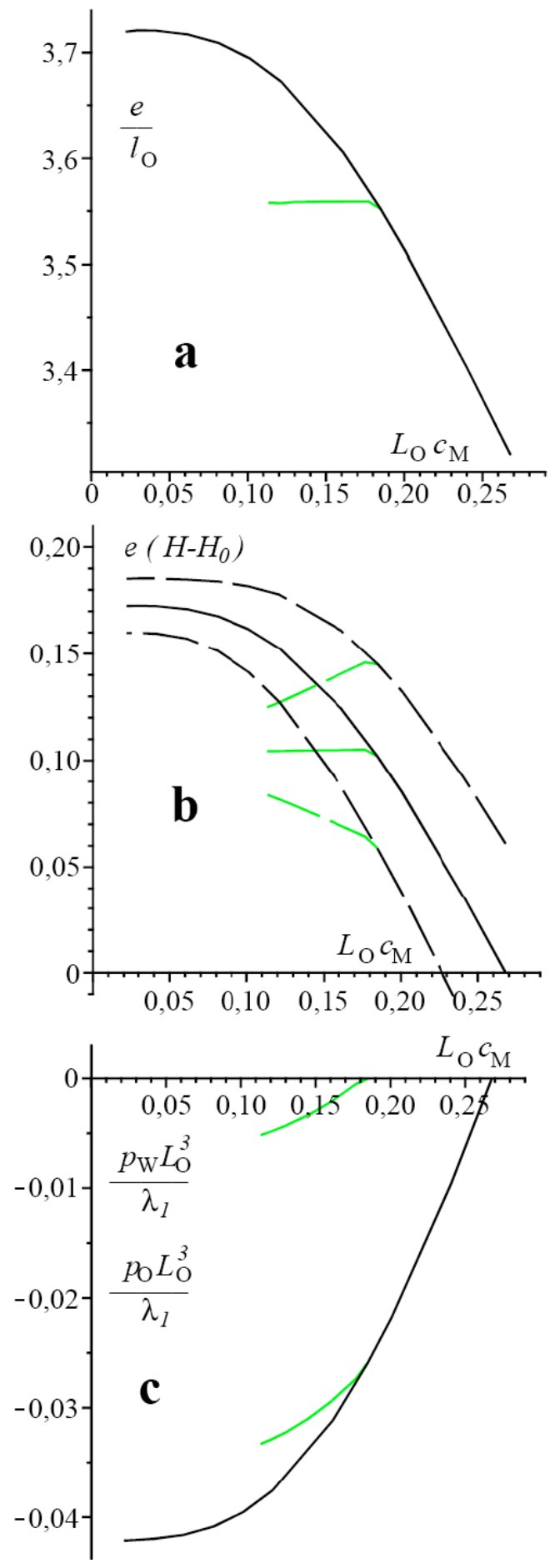

Fig. (18). Oil volume and water volume constraint (green). Dependence on $L_{\mathrm{O}} c_{\mathrm{M}} \cdot \mathbf{a}: e / l_{\mathrm{O}} \cdot \mathbf{b}: e\left(H-H_{0}\right) \cdot \mathbf{c}: p_{\mathrm{O}} L_{\mathrm{O}}^{3} / \lambda_{1}$ and $p_{\mathrm{W}} L_{\mathrm{O}}^{3} / \lambda_{1}$. The case of an oil volume constraint alone is illustrated in black.

\subsection{Consequences of the Lack of Symmetry Breaking}

We consider the case D of Table $\mathbf{1}\left(v<1, D_{0}=0, H_{0}>0\right)$, which gives rise to Helfrich's quadratic energy density (9). Fig. (19a) shows a tube6 solution with $v=0.9$ and without volume constraints. Since we deal with a non-linear problem, it is not surprising that we encounter another solution, shown in Fig. (19b). The values of the first-mentioned solution can be read off from Figs. (5-11) $(\omega=1)$, those of the second solution are given in brackets: $l_{0} c_{\mathrm{M}}=0.3452(0.3408)$, $e H_{0}=2.560(4.195), e / l_{\mathrm{O}}=7.415(12.310), \bar{w} /\left(\lambda_{1} c_{\mathrm{M}}^{2}\right)=0.1617(0.3059)$, $H / H_{0}=0.838 \pm 0.023(0.694 \pm 0.363), \quad D / H_{0}=0.718 \pm 1.432(1.164 \pm 0.424)$. Each of the two solutions describes a state of equilibrium, but the appertaining stationary values of the energy differ almost by a factor of 2 . The reason is the following one: The values of $H$ and $D$ of the solution with higher energy cover a wide range.

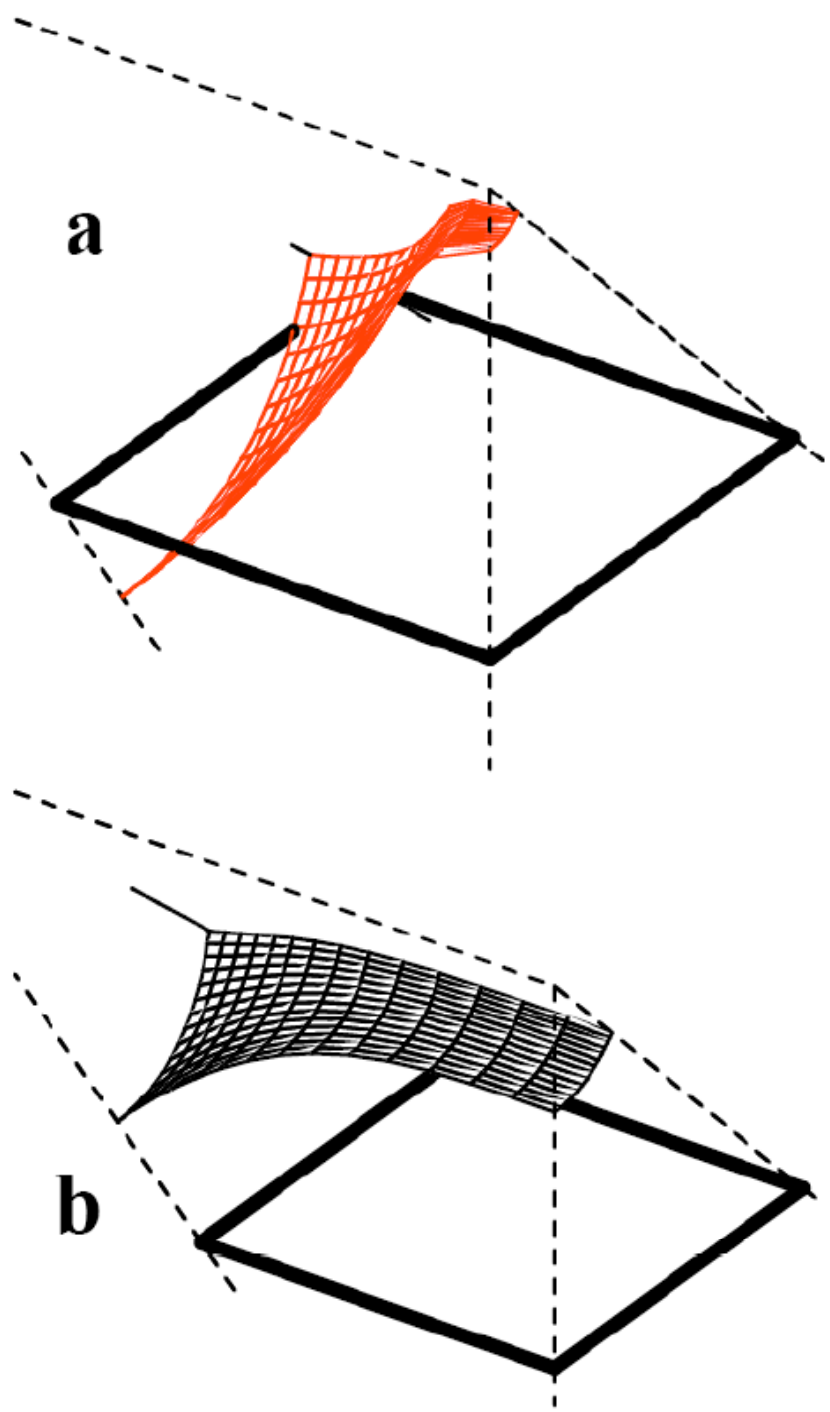

Fig. (19). No volume constraint. Placements of the elementary patch of tube6 in the case $v=0.9, H_{0}>0, D_{0}=0$. a: Solution with minimal energy. $\mathbf{b}$ : Other equilibrium solution. 
The mean curvature $H$ of the solution with the lower energy is more homogeneous and nearer to the preferred value $H_{0}$. Moreover, its elementary patch consists of two distinct parts. The difference $D$ of the mean curvatures is rather small in the region on the left-hand side of the picture, so that its shape is almost that of a sphere. On the right-hand side, however, we see a narrow tunnel which connects this sphere-like region with another one on the other side of the plane of symmetry. Only that small region shows giant values of $D$ and hence of the local energy density $w$.

Nevertheless, it is unlikely that a tube solution - and hence a three-phase coexistence in case of tube 6 and tube 4 - is observed at all, since the minimum possible energy $w \equiv 0$ is provided by a sphere with $H=H_{0}$.

In this context, we encounter another problem. The relative error of the defect is $0.002 \%$ with the bracketed solution but more than $6 \%$ with our minimum solution. The reason is surely the high curvature gradient near the tunnel region. But even if we adopt a rectangular mesh $(4 \times 71$ instead of $16 \times 16$ ), we do not succeed in reducing the error. The same is true if the effect of symmetry breaking is present but too small. The numerical studies show that the relative error exceeds $1 \%$ - and hence the results are dubious - if $\omega>0.70, \omega>0.85, \omega>0.80$, with tube6, tube4, and tube3, respectively. On the other hand, in case of tube 3 with $v=1$ and $H_{0}$ almost zero (case $\mathrm{F}$ of Table $\mathbf{1}$ ), the relative error of the defect is really intolerable with a quadratic mesh, but can be considerably brought down with a rectangular one. (If $e H_{0}=0.1$, then the error is $15 \%$ with a $16 \times 16$ mesh, but less than $2 \%$ with a $41 \times 7$ one.)

Till now, we did not apply values of $v$ smaller than 0.9 . Indeed, computational trials with such values were not successful with tubes over a wide range of $\omega$. On the other hand, if there is no symmetry breaking $(\omega=1)$, then results could be obtained down to $v=1 / 3$ in the case without volume constraint. We present the dependence on $v$ in case of tube 6 ; tube4 and tube3 show the same qualitative behaviour. Figs. $(\mathbf{2 0 a}, \mathbf{2 0 b}, \mathbf{2 0 c}, \mathbf{2 0 d})$ give $e H_{0}, \bar{w} /\left(\lambda_{1} c_{\mathrm{M}}^{2}\right)$, $H / H_{0}$, and $D / H_{0}$ as functions of $(1-v) /(1+v)$. Although we have in mind the considerable error of the defect, we must admit that the tendencies look reasonable.

\subsection{The Carrying Behaviour of the Film}

The following example will reveal the internal forces in the presence of an oil pressure due to an oil volume constraint. We discuss tube6 with case $\mathrm{C}$ of Table $\mathbf{1}$ $\left(v=0.9, H_{0} / D_{0}=0.7\right)$. If we choose $h=0-$ as we did till now - and $l_{\mathrm{O}} D_{0}=0.145$ then we obtain a solution with $\bar{H} / D_{0}=0.90$ and $A_{\mathrm{C}} D_{0}^{2}=0.0423$. Now, we want to study the influence of the film thickness $h$. (Note that no such influence exists in the absence of volume constraints.) According to equation (23) of appendix D and with the definition (11) of the defect, we have

$$
l_{\mathrm{O}}(h)=\frac{V_{\mathrm{OC}}}{A_{\mathrm{C}}}=l_{\mathrm{O}}(h=0)-\frac{h}{2}+\bar{H} \frac{h^{2}}{4}+\frac{\pi}{144} \frac{\delta h^{3}}{A_{\mathrm{C}}}
$$

If we retain the placement and hence the values of $l_{\mathrm{O}}(h=0), \bar{H}$, and $A_{\mathrm{C}}$ of the above solution and choose $h D_{0}=0.2$, then this formula yields $l_{\mathrm{O}} D_{0}=0.0581$. So one could think that the only influence of the thickness is a change of the oil length scale. Actually, however, the correct equilibrium solution corresponding to $l_{\mathrm{O}} D_{0}=0.0581$ is somewhat different. Indeed, the placement and even the mean energy density $\bar{w}$ almost remain constant, but the internal forces and the oil pressure change markedly. The effective pressure on the middle surface according to (5) reduces to

$$
p_{n}=-p_{\mathrm{O}}\left(1-\operatorname{tr} \mathbf{C} \frac{h}{2}+\operatorname{det} \mathbf{C} \frac{h^{2}}{4}\right)
$$

and its mean value becomes

$$
\bar{p}_{n}=-p_{\mathrm{O}}\left(1-\bar{H} h-\frac{\pi}{24} \frac{\delta h^{2}}{A_{\mathrm{C}}}\right)=-0.7 p_{\mathrm{O}}
$$

i.e. $70 \%$ of the absolute value of the oil pressure. Equations (7) and (4) can be given the dimensionless form

$$
\begin{gathered}
\frac{-\frac{\mathbf{T}: \mathbf{C}}{\bar{p}_{n}}=\frac{\frac{p_{n}}{\bar{p}_{n}}}{\bar{p}_{n}}+\frac{\mathbf{q}_{\mathrm{T}} \cdot \nabla_{\mathrm{T}}}{\bar{p}_{n}}}{=-\frac{w_{n}-\bar{w}}{\bar{p}_{n}} \operatorname{tr} \mathbf{C}-\frac{p_{\mathrm{O}} l_{\mathrm{O}}}{\bar{p}_{n}} \operatorname{tr} \mathbf{C}-\frac{\mathbf{M}: \mathbf{C}^{2}}{\bar{p}_{n}}}
\end{gathered}
$$

The two underlined terms are evaluated at all the points of integration in Fig. (21). We note $p_{n} / \bar{p}_{n} \in[0.78,1.22]$ in contrast to the case $h=0$ where we had the simple result $p_{n} \equiv \bar{p}_{n}=-p_{\mathrm{O}}$. The values of the membrane force contribution $-\mathbf{T}: \mathbf{C}$, however, are at some points smaller and at other points even twice as large as the values of $p_{n}$. It is the difference between the two values that must be compensated by the transverse force contribution $\mathbf{q}_{\mathrm{T}} \cdot \nabla_{\mathrm{T}}$. So membrane forces and transverse forces yield carrying contributions of equal order of magnitude. (If no volume constraint is present, then $p_{n} \equiv 0$, and the two contributions cancel each other, but none of them is generally equal to zero.) This finding demonstrates that the static behaviour of our fluid film differs markedly from that of a membrane without bending stiffness, controlled by equation (8). The contribution of the membrane forces is the sum of the three underbraced components. In our example, the first and second term do not exceed the absolute value 0.16 . So the essential term is the third one, which depends on the local moments.

\subsection{Numeric Problems Caused by Symmetry Breaking}

The elementary patches of each of our tubes possess corner angles of $60^{\circ}$, one in case of tube 3 and tube 6 , two in case of tube4. Six patches of the total tube structure meet at 

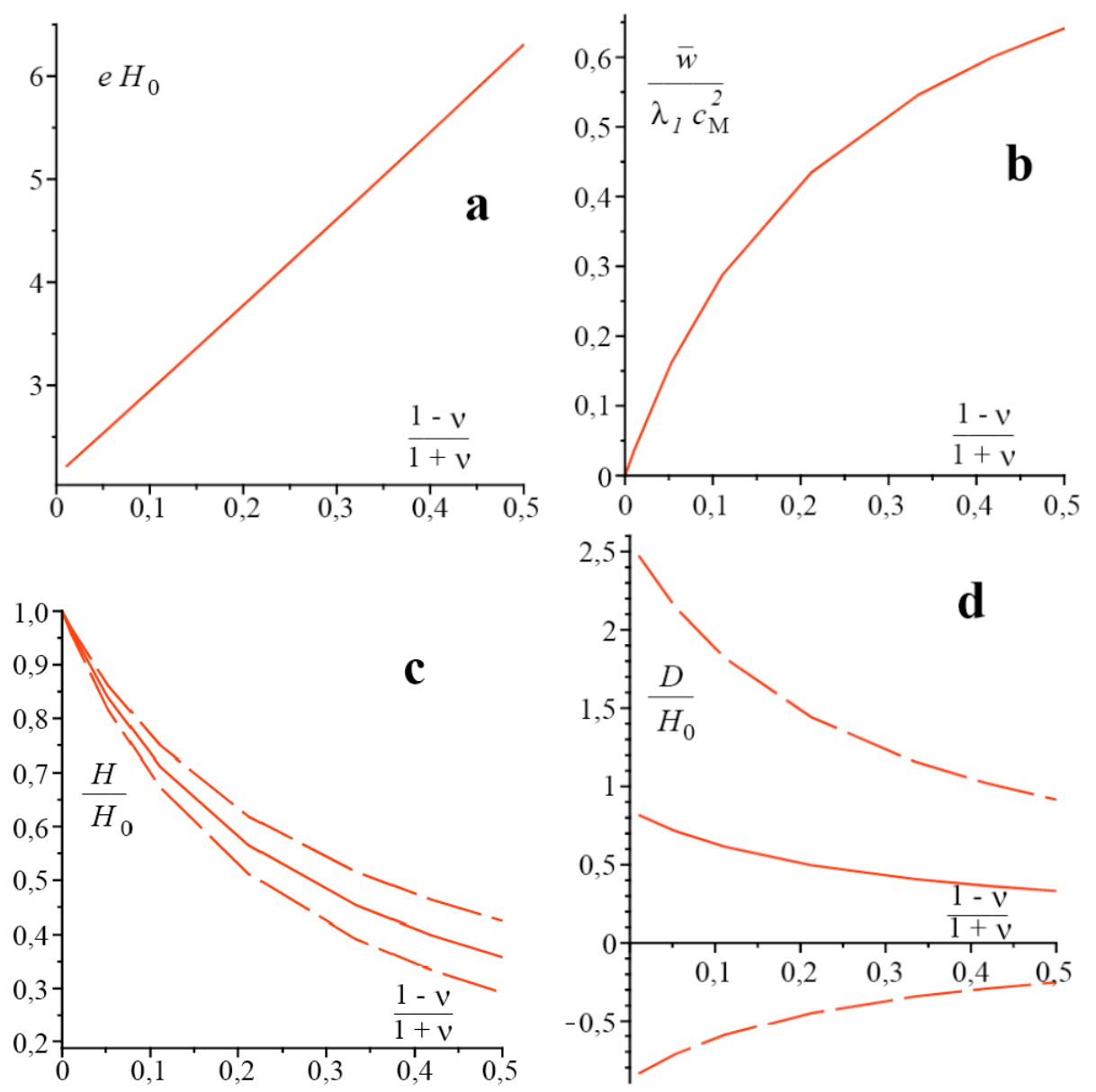

Fig. (20). Influence of the saddle-splay stiffness in the case without symmetry breaking on the following properties: a: $e H_{0} . \mathbf{b}: \bar{w} /\left(\lambda_{1} c_{\mathrm{M}}^{2}\right)$. c: $H / H_{0} . \mathbf{d}: D / H_{0}$.

such a corner. Due to the symmetry requirements, the curvature tensor must be isotropic at that corner point and hence $D=0$ must hold there. If symmetry breaking is present $\left(v<1, D_{0}>0\right)$ then the film will try to adjust its curvature difference $D$ to the preferred difference $D_{0}$, but this is simply impossible at the corner points. The problem becomes more severe with increasing saddle-splay stiffness which means decreasing value of $v$. Consider tube6 without volume constraints and let $H_{0}=D_{0}$ (case C of Table 1). If we choose $v=0.9$, then we obtain a smooth solution. If, however, $v=0.8$, i.e. if the influence of the saddle-splay deformation is nearly duplicated, then the field of the mean curvature $H$ and of the energy density $w$ show oscillatory behaviour near the corner point, which becomes more pronounced with a finer mesh. If even smaller values of $v$ were considered, then not even the convergence to a solution could be achieved. This is surely not a shortcoming of the numerical method but stems from the condition $D=0$ and should also be present in an analytic solution, which unfortunately is not available. After all, if we are only interested in the structure with minimal energy, we know that the cylinder has $w \equiv 0$ under the above setting, so that tubes are insignificant.

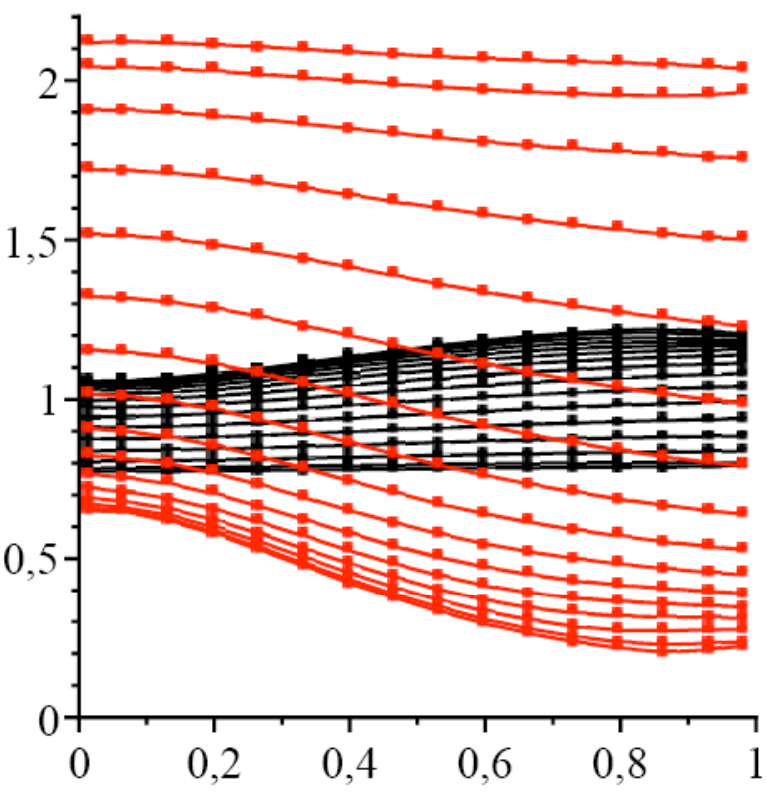

Fig. (21). Values of $-\mathbf{T}: \mathbf{C} / \bar{p}_{n}$ (red) and $p_{n} / \bar{p}_{n}$ (black) at the points of integration. Horizontal axis is $u / r$. Points with equal $v$ are connected. The upper red line and the lower black line correspond to the smallest value $v=0.25$. 


\section{OUTLOOK}

Our investigation was based on continuum mechanics. One might argue that the conformation of a monomolecular film should better be derived from first principles. Indeed, a lot of progress in molecular modelling has been made due to the potential of modern computers. Among the many approaches, based on different idealizations ( $c f$. [15]), the classical density functional theory (DFT) is rather prominent. The authors of [16] succeeded to construct a connection between a quadratic approximation of DFT and the quadratic phenomenological constitutive relation (9) of Helfrich. However, if volume constraints are absent then this relation predicts that the film should have spherical geometry, since this shape minimizes the energy. We infer this result from our Fig. (8), where $D_{0}=0$ corresponds to $\omega=1$. But it is well known that actually bicontinuous structures are observed and form the basis of the three-phase coexistence of a microemulsion with oil and water excesses. They can only be predicted with a non-quadratic constitutive relation, as was already pointed out in $[10,11]$. We obtained symmetry breaking by the introduction of a preferred saddle splay curvature $D_{0}$ in the relation (10). If $H_{0} \ll D_{0}$ and hence $\omega$ near 0 , then Fig. (8) shows us that bicontinuous structures are indeed energetically favorized. It would be highly desirable, to start from a more refined molecular modelling in order to derive a realistic macroscopic description of the dependence of the free energy density on the local curvature of the film. A promising approach seems to be the improved DFT, developed by Shiqi Zhou (see e.g. [17]) which takes into account the formerly discarded terms. Once such a macroscopic law has been derived from first principles, the computation of equilibrium conformations can be done with the numerical method of the paper at present. Such a proceeding surely reduces the computer cost compared with a full molecular modelling of the total conformation. This remains a challenge for further research.

\section{APPENDICES}

\section{A. SOME FACTS FROM DIFFERENTIAL GEOMETRY}

Let $\mathbf{x}(u, v)$ denote the position vector of the middle surface of the film as a function of the surface coordinates $u$ and $v$. The tangent vectors of the surface are obtained by partial differentiation with respect to $u$ and $v$ (denoted by a comma).

$$
\mathbf{g}_{u}=\mathbf{x},_{u}, \quad \mathbf{g}_{v}=\mathbf{x},{ }_{v}
$$

The vector

$$
\mathbf{w}=\mathbf{g}_{u} \times \mathbf{g}_{v}
$$

is then normal to the tangential plane and the unit normal vector of the surface is obtained as

$$
\mathbf{n}=\psi \mathbf{w}
$$

with the abbreviation

$$
\psi=\frac{1}{|\mathbf{w}|}
$$

The cotangent vectors are

$$
\begin{aligned}
& \mathbf{g}^{u}=\psi^{2} \mathbf{g}_{v} \times \mathbf{w}=\psi^{2}\left(g_{v v} \mathbf{g}_{u}-g_{u v} \mathbf{g}_{v}\right) \\
& \mathbf{g}^{v}=\psi^{2} \mathbf{w} \times \mathbf{g}_{u}=\psi^{2}\left(g_{u u} \mathbf{g}_{v}-\mathrm{g}_{u v} \mathbf{g}_{u}\right)
\end{aligned}
$$

with

$$
g_{u u}=\mathbf{g}_{u} \cdot \mathbf{g}_{u}, \quad g_{v v}=\mathbf{g}_{v} \cdot \mathbf{g}_{v}, \quad g_{u v}=\mathbf{g}_{u} \cdot \mathbf{g}_{v}
$$

The oriented surface element is given by

$$
d \mathbf{a}=d A \mathbf{n}=\mathbf{x},{ }_{u} d u \times \mathbf{x},{ }_{v} d v=\mathbf{w} d u d v
$$

and its area by

$$
d A=d \mathbf{a} \cdot \mathbf{n}=\frac{1}{\psi} d u d v
$$

The tensor of curvature is the tangential gradient of the unit normal vector field, endowed with a negative sign.

$$
\begin{aligned}
& \mathbf{C}=-\mathbf{n} \otimes \nabla_{\mathrm{T}}=-\mathbf{n} \otimes\left(\mathbf{g}^{u} \frac{\partial}{\partial u}+\mathbf{g}^{v} \frac{\partial}{\partial v}\right) \\
& =-\mathbf{n},{ }_{u} \otimes \mathbf{g}^{u}-\mathbf{n},{ }_{v} \otimes \mathbf{g}^{v}
\end{aligned}
$$

One representation of the spatial unit tensor is

$$
\mathbf{1}=\mathbf{g}_{u} \otimes \mathbf{g}^{u}+\mathbf{g}_{v} \otimes \mathbf{g}^{v}+\mathbf{n} \otimes \mathbf{n}=\mathbf{1}_{\mathrm{T}}+\mathbf{n} \otimes \mathbf{n}
$$

\section{B. THE EULER-LAGRANGEAN EQUATIONS OF OUR MINIMUM PROBLEM}

Our numerical treatment is based on a discretization of the extended energy which leads to a classical minimum problem with a finite number of unknowns. In this appendix, however, we stay within the continuum formulation, make use of the methods of variational calculus and get interesting insight into the internal forces and torques of a fluid film with bending stiffness. The following exposition is rather condensed. A more detailed presentation within a somewhat broader context can be found in the monograph [11].

The extended energy density (1) can be given the form

$$
\begin{aligned}
& E=n\left(\Phi_{1}+\Phi_{2}\right)+f+p_{\mathrm{O}} L_{\mathrm{O}}+p_{\mathrm{w}} L_{\mathrm{W}} \\
& +\left(w_{\mathrm{O}}-f\right) \frac{A_{\mathrm{O}}}{A}+\left(w_{\mathrm{W}}-f\right) \frac{A_{\mathrm{W}}}{A}
\end{aligned}
$$

with

$$
\begin{aligned}
& \Phi_{1}=\int_{A_{\mathrm{C}}} w(\mathbf{C}) d A-f \int_{A_{\mathrm{C}}} d A \\
& \Phi_{2}=-p_{\mathrm{O}} V_{\mathrm{O} C}-p_{\mathrm{W}} V_{\mathrm{WC}}
\end{aligned}
$$

We shall denote the variation of a quantity by a superposed dot. A necessary condition for the minimum of the bending energy under the given constraints is that the variation of $\Phi_{1}+\Phi_{2}$ vanishes under any change of the placement of the film.

First, we need the variation of some geometric objects. So we get 


$$
\begin{aligned}
& \dot{\mathbf{g}}^{\alpha}=\dot{\mathbf{g}}^{\alpha} \cdot \mathbf{1}=\left(\dot{\mathbf{g}}^{\alpha} \cdot \mathbf{g}_{u}\right) \mathbf{g}^{u}+\left(\dot{\mathbf{g}}^{\alpha} \cdot \mathbf{g}_{v}\right) \mathbf{g}^{v}+\left(\dot{\mathbf{g}}^{\alpha} \cdot \mathbf{n}\right) \mathbf{n} \\
& =-\left(\mathbf{g}^{\alpha} \cdot \dot{\mathbf{g}}_{u}\right) \mathbf{g}^{u}-\left(\mathbf{g}^{\alpha} \cdot \dot{\mathbf{g}}_{v}\right) \mathbf{g}^{v}-\left(\mathbf{g}^{\alpha} \cdot \dot{\mathbf{n}}\right) \mathbf{n} \\
& =-\mathbf{g}^{\alpha} \cdot\left(\dot{\mathbf{g}}_{u} \otimes \mathbf{g}^{u}+\dot{\mathbf{g}}_{v} \otimes \mathbf{g}^{v}+\dot{\mathbf{n}} \otimes \mathbf{n}\right) \\
& =-\mathbf{g}^{\alpha} \cdot\left(\dot{\mathbf{x}} \otimes \nabla_{\mathrm{T}}+\dot{\mathbf{n}} \otimes \mathbf{n}\right), \quad \alpha=u, v
\end{aligned}
$$

and

$$
\begin{aligned}
& \dot{\mathbf{n}}=\dot{\mathbf{n}} \cdot \mathbf{1}=\left(\dot{\mathbf{n}} \cdot \mathbf{g}_{u}\right) \mathbf{g}^{u}+\left(\dot{\mathbf{n}} \cdot \mathbf{g}_{v}\right) \mathbf{g}^{v}+(\dot{\mathbf{n}} \cdot \mathbf{n}) \mathbf{n} \\
& =-\left(\mathbf{n} \cdot \dot{\mathbf{g}}_{u}\right) \mathbf{g}^{u}-\left(\mathbf{n} \cdot \dot{\mathbf{g}}_{v}\right) \mathbf{g}^{v} \\
& =-\mathbf{n} \cdot\left(\dot{\mathbf{g}}_{u} \otimes \mathbf{g}^{u}+\dot{\mathbf{g}}_{v} \otimes \mathbf{g}^{v}\right)=-\mathbf{n} \cdot\left(\dot{\mathbf{x}} \otimes \nabla_{\mathrm{T}}\right)
\end{aligned}
$$

where we used the fact that the variation of the constant values

$$
\begin{array}{ll}
\mathbf{g}^{\alpha} \cdot \mathbf{g}_{u}=\delta_{u}^{\alpha} & \mathbf{g}^{\alpha} \cdot \mathbf{g}_{v}=\delta_{v}^{\alpha} \\
\mathbf{g}^{\alpha} \cdot \mathbf{n}=0 & \mathbf{g}_{\alpha} \cdot \mathbf{n}=0 \quad \mathbf{n} \cdot \mathbf{n}=1
\end{array}
$$

vanishes. (Note that Kronecker's $\delta$ is either 1 or 0 .) We also have

$$
\begin{aligned}
& \dot{\mathbf{C}}=-\dot{\mathbf{n}},{ }_{u} \otimes \mathbf{g}^{u}-\dot{\mathbf{n}},{ }_{v} \otimes \mathbf{g}^{v}-\mathbf{n},{ }_{u} \otimes \dot{\mathbf{g}}^{u}-\mathbf{n},{ }_{v} \otimes \dot{\mathbf{g}}^{v} \\
& =-\dot{\mathbf{n}} \otimes \nabla_{\mathrm{T}}+\left(\mathbf{n} \otimes \nabla_{\mathrm{T}}\right) \cdot\left(\dot{\mathbf{x}} \otimes \nabla_{\mathrm{T}}+\dot{\mathbf{n}} \otimes \mathbf{n}\right)
\end{aligned}
$$

and hence

$$
\dot{\mathbf{C}} \cdot \mathbf{1}_{\mathrm{T}}=-\dot{\mathbf{n}} \otimes \nabla_{\mathrm{T}}-\mathbf{C} \cdot\left(\dot{\mathbf{x}} \otimes \nabla_{\mathrm{T}}\right)
$$

Finally,

$$
\dot{\overline{d \mathbf{a}}}=\dot{\overline{d A}} \mathbf{n}+d A \dot{\mathbf{n}}=\left(\dot{\mathbf{x}}_{, u} \times \mathbf{x},,_{v}+\mathbf{x},{ }_{u} \times \dot{\mathbf{x}}, v\right) d u d v
$$

and hence

$$
\begin{aligned}
& =\psi\left(\dot{\mathbf{x}},{ }_{u} \cdot\left(\mathbf{x},,_{v} \times \mathbf{w}\right)+\left(\mathbf{w} \times \mathbf{x},{ }_{u}\right) \cdot \dot{\mathbf{x}},{ }_{v}\right) d u d v \\
& =\left(\dot{\mathbf{x}},_{u} \cdot \mathbf{g}^{u}+\dot{\mathbf{x}},,_{v} \cdot \mathbf{g}^{v}\right) \frac{1}{\psi} d u d v \\
& =\dot{\mathbf{x}} \cdot \nabla_{\mathrm{T}} d A=\mathbf{1}_{\mathrm{T}}:\left(\dot{\mathbf{x}} \otimes \nabla_{\mathrm{T}}\right) d A
\end{aligned}
$$

If $h$ is the constant thickness of the film, then the surface elements on the oil-sided and the water-sided boundary are given by (note that the normal vector of the middle surface is oriented towards the oil side)

$$
d A_{\mathrm{O} / W}=\left(1 \mp \operatorname{tr} \mathbf{C} \frac{h}{2}+\operatorname{det} \mathbf{C} \frac{h^{2}}{4}\right) d A
$$

A variation of the placement of the surface yields

$$
\begin{aligned}
& \dot{\Phi}_{2}=-p_{\mathrm{O}} \dot{V}_{\mathrm{O} C}-p_{\mathrm{W}} \dot{V}_{\mathrm{W} C} \\
& =p_{\mathrm{O}} \int_{A_{\mathrm{O} C}} \dot{\mathbf{x}} \cdot \mathbf{n} d A_{\mathrm{O}}-p_{\mathrm{W}} \int_{A_{\mathrm{W} C}} \dot{\mathbf{x}} \cdot \mathbf{n} d A_{\mathrm{W}} \\
& =-\int_{A_{\mathrm{C}}} \dot{\mathbf{x}} \cdot \mathbf{n} p_{n} d A
\end{aligned}
$$

with the abbreviation $p_{n}$ according to (5).
The energy density $w$ is an isotropic function of the local curvature tensor $\mathbf{C}$, which is a symmetric tensor. Its derivative, endowed with a negative sign and called tensor of moments,

$$
\mathbf{M}=-\frac{\partial w}{\partial \mathbf{C}}
$$

is also a symmetric tensor in the tangential plane, which has the same principal axes as $\mathbf{C}$ and hence commutes with $\mathbf{C}$ so that $\mathbf{C} \cdot \mathbf{M}=\mathbf{M} \cdot \mathbf{C}$ is a symmetric tensor, too. Therefore,

$$
\begin{aligned}
& \dot{w}=\frac{\partial w}{\partial \mathbf{C}}: \dot{\mathbf{C}}=-\mathbf{M}: \dot{\mathbf{C}} \\
& =-\left(\mathbf{M} \cdot \mathbf{1}_{\mathrm{T}}\right): \dot{\mathbf{C}}=-\mathbf{M}:\left(\dot{\mathbf{C}} \cdot \mathbf{1}_{\mathrm{T}}\right) \\
& =\mathbf{M}:\left(\dot{\mathbf{n}} \otimes \nabla_{\mathrm{T}}+\mathbf{C} \cdot\left(\dot{\mathbf{x}} \otimes \nabla_{\mathrm{T}}\right)\right) \\
& =\mathbf{M}:\left(\dot{\mathbf{n}} \otimes \nabla_{\mathrm{T}}\right)+(\mathbf{C} \cdot \mathbf{M}):\left(\dot{\mathbf{x}} \otimes \nabla_{\mathrm{T}}\right)
\end{aligned}
$$

For brevity, we also introduce the symmetric tensor of membrane forces

$$
\mathbf{T}=(w-f) \mathbf{1}_{\mathrm{T}}+\mathbf{C} \cdot \mathbf{M}
$$

and find

$$
\begin{aligned}
& \dot{\Phi}_{1}=\int_{A_{\mathrm{C}}}[\dot{w} d A+(w-f) \dot{d A}] \\
& =\int_{A_{\mathrm{C}}}\left[\mathbf{T}:\left(\dot{\mathbf{x}} \otimes \nabla_{\mathrm{T}}\right)+\mathbf{M}:\left(\dot{\mathbf{n}} \otimes \nabla_{\mathrm{T}}\right)\right] d A
\end{aligned}
$$

The two terms represent the internal power of the membrane forces and of the moments, respectively, on a stretching and bending of the surface. We saw in (14) that the fields $\dot{\mathbf{n}}$ and $\dot{\mathbf{x}}$ are not independent, so that

$$
0=\int_{A_{\mathrm{C}}}\left[\dot{\mathbf{n}}+\mathbf{n} \cdot\left(\dot{\mathbf{x}} \otimes \nabla_{\mathrm{T}}\right)\right] \cdot \mathbf{q}_{\mathrm{T}} d A
$$

is valid for any choice of the tangential vector field $\mathbf{q}_{\mathrm{T}}$. We interpret $\mathbf{q}_{\mathrm{T}}$, called operator of transverse forces, as a Lagrangean parameter, add (16) to (15), apply integration by parts with the help of the divergence theorem of curved surfaces and arrive at the condition

$$
\begin{aligned}
& 0=\dot{\Phi}_{1}+\dot{\Phi}_{2} \\
& =\oint\left[\dot{\mathbf{x}} \cdot\left(\mathbf{T}+\mathbf{n} \otimes \mathbf{q}_{\mathrm{T}}\right) \cdot \mathbf{e}+\dot{\mathbf{n}} \cdot \mathbf{M} \cdot \mathbf{e}\right] d s \\
& -\int_{A_{\mathrm{C}}}\left(\dot{\mathbf{x}} \cdot\left[\left(\mathbf{T}+\mathbf{n} \otimes \mathbf{q}_{\mathrm{T}}\right) \cdot \nabla_{\mathrm{T}}+p_{n} \mathbf{n}\right]\right. \\
& \left.+\dot{\mathbf{n}} \cdot \mathbf{1}_{\mathrm{T}} \cdot\left(\mathbf{M} \cdot \nabla_{\mathrm{T}}-\mathbf{q}_{\mathrm{T}}\right)\right) d A
\end{aligned}
$$

(We made use of $\dot{\mathbf{n}}=\dot{\mathbf{n}} \cdot \mathbf{1}_{\mathrm{T}}$ because of $\dot{\mathbf{n}} \cdot \mathbf{n}=0$.)

We first concentrate on the line integral, which is evaluated along the boundaries of a surface patch. The line element of one boundary shall be written $d \mathbf{x}=\mathbf{g} d s$, while the unit vector $\mathbf{e}$ within the tangential plane, defined by 


$$
\mathbf{e}=\mathbf{g} \times \mathbf{n}
$$

is normal to the boundary unit vector $\mathbf{g}$. We are interested in the case, where all of the boundaries lie on planes of symmetry of the total surface structure. So e must coincide with the constant unit normal vector $\mathbf{e}_{\mathrm{s}}$ of such a plane of symmetry, and we have the geometric boundary condition

$$
\begin{aligned}
& \mathbf{e}_{\mathrm{S}} \cdot \mathbf{n}=0 \quad \Rightarrow \\
& 0=\frac{d}{d s}\left(\mathbf{e}_{\mathrm{S}} \cdot \mathbf{n}\right)=\mathbf{e}_{\mathrm{S}} \cdot\left(\mathbf{n} \otimes \nabla_{T}\right) \cdot \mathbf{g}=-\mathbf{e}_{\mathrm{S}} \cdot \mathbf{C} \cdot \mathbf{g}
\end{aligned}
$$

This means that $\mathbf{e}$ and $\mathbf{g}$ are proper vectors of $\mathbf{C}$ and hence also of $\mathbf{M}$ and $\mathbf{T}$. Therefore, the boundary is a line of principal curvature and the shearing membrane force and the twisting moment must vanish there.

$$
\begin{aligned}
& \mathbf{g} \cdot \mathbf{T} \cdot \mathbf{e}=0, \quad \mathbf{g} \cdot \mathbf{M} \cdot \mathbf{e}=0 \Rightarrow \\
& \mathbf{T} \cdot \mathbf{e}=(\mathbf{e} \cdot \mathbf{T} \cdot \mathbf{e}) \mathbf{e}, \quad \mathbf{M} \cdot \mathbf{e}=(\mathbf{e} \cdot \mathbf{M} \cdot \mathbf{e}) \mathbf{e}
\end{aligned}
$$

The boundary integral becomes

$$
\oint\left[\underline{(\dot{\mathbf{x}} \cdot \mathbf{e})}(\mathbf{e} \cdot \mathbf{T} \cdot \mathbf{e})+(\dot{\mathbf{x}} \cdot \mathbf{n})\left(\mathbf{q}_{T} \cdot \mathbf{e}\right)+\underline{(\dot{\mathbf{n}} \cdot \mathbf{e})}(\mathbf{e} \cdot \mathbf{M} \cdot \mathbf{e})\right] d s
$$

The boundary points $\mathbf{x}$ and the normal $\mathbf{n}$ must remain in the plane of symmetry during a variation of the placement and hence the underlined terms are zero. So the normal membrane force $\mathbf{e} \cdot \mathbf{T} \cdot \mathbf{e}$ and the bending moment $\mathbf{e} \cdot \mathbf{M} \cdot \mathbf{e}$ are not restricted on the boundaries. The remaining power of the transverse force $\left(\mathbf{q}_{T} \cdot \mathbf{e}\right) \mathbf{n}$ can only be zero for arbitrary functions $\dot{\mathbf{x}} \cdot \mathbf{n}$ if the transverse force vanishes along the boundary, and the natural boundary condition is therefore

$$
\mathbf{q}_{T} \cdot \mathbf{e}=0
$$

The area integrals remain to be discussed. Since $\dot{\mathbf{x}}$ is an arbitrary spatial vector field and $\dot{\mathbf{n}}$ an arbitrary tangential vector field, the following Euler-Lagrangean differential equations must be valid

$$
\left(\mathbf{T}+\mathbf{n} \otimes \mathbf{q}_{\mathrm{T}}\right) \cdot \nabla_{\mathrm{T}}+p_{n} \mathbf{n}=\mathbf{0}
$$

and

$$
\mathbf{1}_{\mathrm{T}} \cdot\left(\mathbf{M} \cdot \nabla_{\mathrm{T}}\right)-\mathbf{q}_{\mathrm{T}}=\mathbf{0}
$$

The first of these field equations expresses the equilibrium of the internal and external forces in the three directions of space and the latter the equilibrium of moments around any axis within the tangential plane.

Regarding first the equilibrium of forces within the tangential plane and eliminating the operator of transverse forces, we find

$$
\mathbf{1}_{\mathrm{T}} \cdot\left(\left[\mathbf{T}+\mathbf{n} \otimes \mathbf{1}_{\mathrm{T}} \cdot\left(\mathbf{M} \cdot \nabla_{\mathrm{T}}\right)\right] \cdot \nabla_{\mathrm{T}}\right)=\mathbf{0}
$$

Now, the fields $\mathbf{T}$ and $\mathbf{M}$ depend on the field of curvature $\mathbf{C}$ and the constant $f$. The following can be proved ( $c f$. [11] for a proof within a broader context).

Theorem: The left-hand side of equation (18) is identically zero for any placement of the surface and any choice of $f$.
So the tangential equilibrium of forces is identically satisfied and need not be cared for.

This result has its origin in the peculiar dependence of the membrane forces $\mathbf{T}$ of a fluid film on the curvature. It is in sharp contrast to the behaviour of solid shells, the membrane forces of which depend essentially on the stretches of the middle surface.

So we may restrict our attention to the equilibrium of forces in the normal direction

$$
\begin{aligned}
& 0=\mathbf{n} \cdot\left(\left(\mathbf{T}+\mathbf{n} \otimes \mathbf{q}_{\mathrm{T}}\right) \cdot \nabla_{\mathrm{T}}+p_{n} \mathbf{n}\right) \\
& =\underline{(\mathbf{n} \cdot \mathbf{T})} \cdot \nabla_{\mathrm{T}}-\mathbf{T}:\left(\mathbf{n} \otimes \nabla_{\mathrm{T}}\right) \\
& +(\mathbf{n} \cdot \mathbf{n})\left(\mathbf{q}_{\mathrm{T}} \cdot \nabla_{\mathrm{T}}\right)+\underline{\mathbf{n} \cdot\left(\mathbf{n} \otimes \nabla_{\mathrm{T}}\right)} \cdot \mathbf{q}_{\mathrm{T}}+p_{n}
\end{aligned}
$$

where the underlined terms vanish, and arrive at

$$
\mathbf{T}: \mathbf{C}+\mathbf{q}_{\mathrm{T}} \cdot \nabla_{\mathrm{T}}=-p_{n}
$$

Making use of vector and tensor components, the equilibrium conditions (17) and (19) - our former equations (3) and (4) - may be written

$$
\begin{aligned}
& \sum_{\alpha=u, v}\left(m_{\beta}^{\alpha},_{\alpha}+\sum_{\gamma=u, v}\left[m_{\beta}^{\gamma}\left(\mathbf{g}^{\alpha} \cdot \mathbf{x},_{\alpha \gamma}\right)\right.\right. \\
& \left.\left.-m_{\gamma}^{\alpha}\left(\mathbf{g}^{\gamma} \cdot \mathbf{x},_{\alpha \beta}\right)\right]\right)-q_{\beta}=0 \quad \beta=u, v \\
& \sum_{\alpha=u, v}\left(\sum_{\gamma=u, v}\left[t_{\alpha}^{\gamma} c_{\gamma}^{\alpha}+q^{\gamma}\left(\mathbf{g}^{\alpha} \cdot \mathbf{x},_{\alpha \gamma}\right)\right]+q^{\alpha},_{\alpha}\right)=-p_{n}
\end{aligned}
$$

Next we render the description of a surface more precise by introducing a reference plane and characterizing a point of the curved surface by its deviation from that plane in the following manner.

$$
\begin{aligned}
& \mathbf{x}(u, v)=\mathbf{x}^{\mathrm{R}}(u, v)+\zeta(u, v) \mathbf{m}(u, v) \\
& =e\left(\tilde{\mathbf{x}}^{R}(u, v)+\theta(u, v) \mathbf{m}(u, v)\right)
\end{aligned}
$$

We may think of $\zeta \mathbf{m}=e \theta \mathbf{m}$ as a displacement from the reference position $\mathbf{x}^{\mathrm{R}}=e \tilde{\mathbf{x}}^{\mathrm{R}}$ to the actual position $\mathbf{x}$. The introduction of the characteristic length $e$ of the structure and the dimensionless quantities $\tilde{\mathbf{x}}^{\mathrm{R}}$ and $\theta$ allows a separation of magnitude and shape of the placement of the structure, and $\mathbf{m}$ denotes a field of unit vectors, called directors. The above representation is common in the treatment of solid shells, where the unknown vector field $\zeta \mathbf{m}$ has to be computed. Its variation, called the virtual displacement, is then the vector field $\dot{\zeta} \mathbf{m}+\zeta \dot{\mathbf{m}}$, the three components of which ensure the equilibrium of forces in the three directions of space.

However, we are in a much better situation. We may prescribe the director field $\mathbf{m}(u, v)$ and only regard the scalar field $\zeta(u, v)$ as unknown. This can be seen in two ways. First, the placement of the surface and hence its curvature are fully determined by this mode of procedure, and it is only the curvature that enters the energy. Second, 
the variation of the displacement reduces to $\dot{\zeta} \mathbf{m}$ which only ensures local equilibrium in the direction of $\mathbf{m}$. But if we exclude the possibility $\mathbf{m} \cdot \mathbf{n}=0$, then the equilibrium condition in the direction of $\mathbf{n}$ - which is the only one to be regarded with fluid films - is also satisfied. Shortly speaking, any point of a solid shell has three degrees of freedom while any point of our fluid film with bending stiffness needs only one. On the other hand, this is the reason why the wealth of software for the treatment of solid shells is not applicable to our topic.

\section{MATHEMATICAL DESCRIPTION OF THE STRUCTURES}

Our numerical investigation concerns the classes of tube3, tube4, and tube6. Additionally, we may treat spheres and cylinders numerically in order to judge the performance of the numerical method, since a comparison with exact solutions is possible.

As is detailed in the following, we choose four planes of symmetry of the structure. They enclose a region that is finite in the case of tube 4 and tube 6 and infinite in the case of sphere, cylinder, and tube 3 . We call that region the cell and the part of the surface contained in it the patch. The whole space and the totality of the structure are obtained by repeated mirror reflections through the planes of symmetry.

We introduce an orthonormal basis $\left\{\mathbf{e}_{1}, \mathbf{e}_{2}, \mathbf{e}_{3}\right\}$. Next, we choose a plane quadrilateral reference patch located in the cell, normal to $\mathbf{e}_{3}$, and bounded by the four planes of symmetry. The position vectors of its four corners are denoted by $\mathbf{x}_{00}^{\mathrm{R}}, \mathbf{x}_{r 0}^{\mathrm{R}}, \mathbf{x}_{0 s}^{\mathrm{R}}, \mathbf{x}_{r s}^{\mathrm{R}}$, and the straight lines of intersection of two planes of symmetry at these corners are characterized by the unit vectors $\mathbf{m}_{00}, \mathbf{m}_{r 0}, \mathbf{m}_{0 s}, \mathbf{m}_{r s}$. We also introduce

$$
\tilde{\mathbf{m}}=\frac{\mathbf{m}}{\mathbf{m} \cdot \mathbf{e}_{3}} \quad \text { with } \quad \tilde{\mathbf{m}} \cdot \mathbf{e}_{3}=1
$$

Bilinear interpolation of $\mathbf{x}^{\mathrm{R}}$ and $\tilde{\mathbf{m}}$, respectively, in the domain $u \in[0, r], v \in[0, s]$ yields

$$
\begin{aligned}
& \mathbf{x}^{\mathrm{R}}(u, v)=\mathbf{x}_{00}^{\mathrm{R}}\left(1-\frac{u}{r}\right)\left(1-\frac{v}{s}\right)+\mathbf{x}_{r 0}^{\mathrm{R}} \frac{u}{r}\left(1-\frac{v}{s}\right) \\
& +\mathbf{x}_{0 s}^{\mathrm{R}}\left(1-\frac{u}{r}\right) \frac{v}{s}+\mathbf{x}_{r s}^{\mathrm{R}} \frac{u}{r} \frac{v}{s}
\end{aligned}
$$

and

$$
\begin{aligned}
& \tilde{\mathbf{m}}(u, v)=\tilde{\mathbf{m}}_{00}\left(1-\frac{u}{r}\right)\left(1-\frac{v}{s}\right)+\tilde{\mathbf{m}}_{r 0} \frac{u}{r}\left(1-\frac{v}{s}\right) \\
& +\tilde{\mathbf{m}}_{0 s}\left(1-\frac{u}{r}\right) \frac{v}{s}+\tilde{\mathbf{m}}_{r s} \frac{u}{r} \frac{v}{s}
\end{aligned}
$$

The director field $\mathbf{m}$ is then obtained by

$$
\mathbf{m}(u, v)=\frac{\tilde{\mathbf{m}}(u, v)}{|\tilde{\mathbf{m}}(u, v)|}
$$

and is an irrational function of $u$ and $v$.

Our quadrilateral curved patch is bounded by four geodesics. Therefore the Gauss-Bonnet theorem reads with the definition (11) of the defect $\delta$ of the patch -

$$
\frac{\pi}{6} \delta=-\int_{A_{\mathrm{C}}} K d A=2 \pi-\sum_{i=1}^{4} \alpha_{i}
$$

and connects the integral over the Gaussian curvature $K$ with the difference of the sum of inner corner angles of a curved and a plane quadrangle. But the corner angles $\alpha_{i}$ are easily obtained as the angles between the planes of symmetry at the corners.

The reference patch divides the total volume of the cell into an oil-sided part $V_{\mathrm{OC}}^{\mathrm{R}}$ and a water-sided part $V_{\mathrm{wC}}^{\mathrm{R}}$. If a cylinder, sphere or tube 3 surface is discussed, we select the case where oil is enclosed.

\section{C.1. Cylinder}

The quadrilateral reference patch is a square. At its corners, the position vectors are

$$
\begin{aligned}
& \mathbf{x}_{00}^{\mathrm{R}}=\mathbf{0}, \quad \mathbf{x}_{r 0}^{\mathrm{R}}=a \mathbf{e}_{1} \\
& \mathbf{x}_{0 s}^{\mathrm{R}}=a \mathbf{e}_{2}, \quad \mathbf{x}_{r s}^{\mathrm{R}}=a\left(\mathbf{e}_{1}+\mathbf{e}_{2}\right)
\end{aligned}
$$

and the directors are

$$
\begin{aligned}
& \mathbf{m}_{00}=\mathbf{e}_{3}=\tilde{\mathbf{m}}_{00}, \quad \mathbf{m}_{r 0}=\frac{1}{\sqrt{2}}\left(\mathbf{e}_{3}-\mathbf{e}_{1}\right)=\frac{1}{\sqrt{2}} \tilde{\mathbf{m}}_{r 0}, \\
& \mathbf{m}_{0 s}=\mathbf{e}_{3}=\tilde{\mathbf{m}}_{0 s}, \quad \mathbf{m}_{r s}=\frac{1}{\sqrt{2}}\left(\mathbf{e}_{3}-\mathbf{e}_{1}\right)=\frac{1}{\sqrt{2}} \tilde{\mathbf{m}}_{r s}
\end{aligned}
$$

Eight patches form a finite section of the full cylinder. Each of the corner angles of a patch is $90^{\circ}$, and hence the defect is $\delta=0$. We have $V_{\mathrm{O} C}^{\mathrm{R}}=a^{3} / 2$ and $V_{\mathrm{W} C}^{\mathrm{R}}=\infty$. The length $a$ is arbitrary but fixed while the value of the characteristic length $e=4 R$ of the cylinder is an outcome of the computation.

\section{C.2. Sphere}

The quadrilateral reference patch is a square. At its corners, the position vectors are

$$
\begin{aligned}
& \mathbf{x}_{00}^{\mathrm{R}}=\mathbf{0}, \quad \mathbf{x}_{r 0}^{\mathrm{R}}=a \mathbf{e}_{1} \\
& \mathbf{x}_{0 s}^{\mathrm{R}}=a \mathbf{e}_{2}, \quad x_{r s}^{\mathrm{R}}=a\left(\mathbf{e}_{1}+\mathbf{e}_{2}\right)
\end{aligned}
$$

and the directors are

$$
\begin{aligned}
& \mathbf{m}_{00}=\mathbf{e}_{3}=\tilde{\mathbf{m}}_{00} \\
& \mathbf{m}_{r 0}=\frac{1}{\sqrt{2}}\left(\mathbf{e}_{3}-\mathbf{e}_{1}\right)=\frac{1}{\sqrt{2}} \tilde{\mathbf{m}}_{r 0},
\end{aligned}
$$




$$
\begin{aligned}
& \mathbf{m}_{0 s}=\frac{1}{\sqrt{2}}\left(\mathbf{e}_{3}-\mathbf{e}_{2}\right)=\frac{1}{\sqrt{2}} \tilde{\mathbf{m}}_{0 s} \\
& \mathbf{m}_{r s}=\frac{1}{\sqrt{3}}\left(\mathbf{e}_{3}-\mathbf{e}_{1}-\mathbf{e}_{2}\right)=\frac{1}{\sqrt{3}} \tilde{\mathbf{m}}_{r s}
\end{aligned}
$$

24 cells fill the whole space and the enclosed 24 patches form the entire sphere surface. The corner angles of a patch are $90^{\circ}, 90^{\circ}, 90^{\circ}, 120^{\circ}$, and hence the defect is $\delta=-1$. We have $V_{\mathrm{O} C}^{\mathrm{R}}=a^{3} / 3$ and $V_{\mathrm{W} C}^{\mathrm{R}}=\infty$. The length $a$ is arbitrary but fixed while the value of the characteristic length $e=2 R$ of the sphere is an outcome of the computation.

\section{C.3. Tube6}

The quadrilateral reference patch is a square. At its corners, the position vectors are

$$
\begin{aligned}
& \mathbf{x}_{00}^{\mathrm{R}}=\mathbf{0}, \quad \mathbf{x}_{r 0}^{\mathrm{R}}=\frac{e}{4} \mathbf{e}_{1} \\
& \mathbf{x}_{0 s}^{\mathrm{R}}=\frac{e}{4} \mathbf{e}_{2}, \quad \mathbf{x}_{r s}^{\mathrm{R}}=\frac{e}{4}\left(\mathbf{e}_{1}+\mathbf{e}_{2}\right)
\end{aligned}
$$

and the directors are

$$
\begin{aligned}
& \mathbf{m}_{00}=\mathbf{e}_{3}=\tilde{\mathbf{m}}_{00} \\
& \mathbf{m}_{r 0}=\frac{1}{\sqrt{2}}\left(\mathbf{e}_{3}-\mathbf{e}_{1}\right)=\frac{1}{\sqrt{2}} \tilde{\mathbf{m}}_{r 0}, \\
& \mathbf{m}_{0 s}=\frac{1}{\sqrt{2}}\left(\mathbf{e}_{3}+\mathbf{e}_{2}\right)=\frac{1}{\sqrt{2}} \tilde{\mathbf{m}}_{0 s}, \\
& \mathbf{m}_{r s}=\frac{1}{\sqrt{3}}\left(\mathbf{e}_{3}-\mathbf{e}_{1}+\mathbf{e}_{2}\right)=\frac{1}{\sqrt{3}} \tilde{\mathbf{m}}_{r s}
\end{aligned}
$$

One vertex of the skeletal graph of the oil-sided tube has co-ordinates $(0, e / 2, e / 4)$, thus being located at one corner of our cell, where three planes of symmetry meet. The six edges of the graph starting from that vertex are characterized by the unit vectors $\pm \mathbf{e}_{1}, \pm \mathbf{e}_{2}, \pm \mathbf{e}_{3}$. The corner angles of a patch are $90^{\circ}, 90^{\circ}, 90^{\circ}, 60^{\circ}$, and hence the defect is $\delta=1$. We have $V_{\mathrm{OC}}^{\mathrm{R}}=V_{\mathrm{WC}}^{\mathrm{R}}=e^{3} / 96$. The characteristic length $e$ is varied during the computation.

\section{C.4. Tube4}

The quadrilateral reference patch is a parallelogram. At its corners, the position vectors are

$$
\begin{aligned}
& \mathbf{x}_{00}^{\mathrm{R}}=-\frac{e}{3 \sqrt{2}} \mathbf{e}_{1}-\frac{2 e}{3} \mathbf{e}_{2}-\frac{e}{\sqrt{6}} \mathbf{e}_{3} \\
& \mathbf{x}_{r 0}^{\mathrm{R}}=\frac{e}{3 \sqrt{2}} \mathbf{e}_{1}-\frac{5 e}{6} \mathbf{e}_{2}-\frac{e}{\sqrt{6}} \mathbf{e}_{3}, \\
& \mathbf{x}_{0 s}^{\mathrm{R}}=-\frac{e}{3 \sqrt{2}} \mathbf{e}_{1}-\frac{e}{6} \mathbf{e}_{2}-\frac{e}{\sqrt{6}} \mathbf{e}_{3} \\
& \mathbf{x}_{r s}^{\mathrm{R}}=\frac{e}{3 \sqrt{2}} \mathbf{e}_{1}-\frac{e}{3} \mathbf{e}_{2}-\frac{e}{\sqrt{6}} \mathbf{e}_{3}
\end{aligned}
$$

and the directors are

$$
\begin{aligned}
& \mathbf{m}_{00}=\frac{1}{\sqrt{2}}\left(\frac{1}{\sqrt{3}} \mathbf{e}_{1}-\frac{\sqrt{2}}{\sqrt{3}} \mathbf{e}_{2}+\mathbf{e}_{3}\right)=\frac{1}{\sqrt{2}} \tilde{\mathbf{m}}_{00}, \\
& \mathbf{m}_{r 0}=\frac{\sqrt{2}}{\sqrt{3}}\left(-\frac{1}{\sqrt{3}} \mathbf{e}_{1}-\frac{1}{\sqrt{6}} \mathbf{e}_{2}+\mathbf{e}_{3}\right)=\frac{\sqrt{2}}{\sqrt{3}} \tilde{\mathbf{m}}_{r 0}, \\
& \mathbf{m}_{0 s}=\frac{\sqrt{2}}{\sqrt{3}}\left(\frac{1}{\sqrt{3}} \mathbf{e}_{1}+\frac{1}{\sqrt{6}} \mathbf{e}_{2}+\mathbf{e}_{3}\right)=\frac{\sqrt{2}}{\sqrt{3}} \tilde{\mathbf{m}}_{0 s}, \\
& \mathbf{m}_{r s}=\frac{1}{\sqrt{2}}\left(-\frac{1}{\sqrt{3}} \mathbf{e}_{1}+\frac{\sqrt{2}}{\sqrt{3}} \mathbf{e}_{2}+\mathbf{e}_{3}\right)=\frac{1}{\sqrt{2}} \tilde{\mathbf{m}}_{r s}
\end{aligned}
$$

Two vertices of the skeletal graph of the oil-sided tube have co-ordinates $(0,0,0)$ and $(0,-e, 0)$, respectively, each being located at one corner of our cell. The four edges of the graph starting from the vertex at the origin are characterized by the unit vectors $-\mathbf{e}_{2}, \quad\left(-2 \sqrt{2} \mathbf{e}_{1}+\mathbf{e}_{2}\right) / 3$, and $\left(\sqrt{2} \mathbf{e}_{1}+\mathbf{e}_{2}\right) / 3 \pm \sqrt{2 / 3} \mathbf{e}_{3}$. those starting at the other vertex by the opposite vectors. The corner angles of a patch are $90^{\circ}, 60^{\circ}, 90^{\circ}, 60^{\circ}$, and hence the defect is $\delta=2$. We have $V_{\mathrm{OC}}^{\mathrm{R}}=V_{\mathrm{WC}}^{\mathrm{R}}=e^{3} /(9 \sqrt{3})$. The characteristic length $e$ is varied during the computation.

\section{C.5. Tube3}

The quadrilateral reference patch is a trapezoid. At its corners, the position vectors are

$$
\begin{aligned}
& \mathbf{x}_{00}^{\mathrm{R}}=-\frac{e}{2 \sqrt{6}} \mathbf{e}_{1}-\frac{e}{2} \mathbf{e}_{2}-\frac{e}{2 \sqrt{6}} \mathbf{e}_{3} \\
& \mathbf{x}_{r 0}^{\mathrm{R}}=\frac{e}{2 \sqrt{6}} \mathbf{e}_{1}-\frac{e}{2} \mathbf{e}_{2}-\frac{e}{2 \sqrt{6}} \mathbf{e}_{3}, \\
& \mathbf{x}_{0 s}^{\mathrm{R}}=-\frac{e}{2 \sqrt{6}} \mathbf{e}_{1}-\frac{e}{6} \mathbf{e}_{2}-\frac{e}{2 \sqrt{6}} \mathbf{e}_{3} \\
& \mathbf{x}_{r s}^{\mathrm{R}}=\frac{e}{2 \sqrt{6}} \mathbf{e}_{1}-\frac{e}{2 \sqrt{6}} \mathbf{e}_{3}
\end{aligned}
$$

and the directors are

$$
\begin{aligned}
& \mathbf{m}_{00}=\frac{1}{\sqrt{2}}\left(\mathbf{e}_{1}+\mathbf{e}_{3}\right)=\frac{1}{\sqrt{2}} \tilde{\mathbf{m}}_{00}, \\
& \mathbf{m}_{r 0}=\frac{1}{\sqrt{2}}\left(-\mathbf{e}_{1}+\mathbf{e}_{3}\right)=\frac{1}{\sqrt{2}} \tilde{\mathbf{m}}_{r 0}, \\
& \mathbf{m}_{0 s}=\frac{\sqrt{3}}{2 \sqrt{2}}\left(\mathbf{e}_{1}+\frac{\sqrt{2}}{\sqrt{3}} \mathbf{e}_{2}+\mathbf{e}_{3}\right)=\frac{\sqrt{3}}{2 \sqrt{2}} \tilde{\mathbf{m}}_{0 s}, \\
& \mathbf{m}_{r s}=\frac{1}{\sqrt{2}}\left(-\mathbf{e}_{1}+\mathbf{e}_{3}\right)=\frac{1}{\sqrt{2}} \tilde{\mathbf{m}}_{r s}
\end{aligned}
$$

The skeletal graph lies in a plane through the origin with unit normal vector $\left(\mathbf{e}_{3}-\mathbf{e}_{1}\right) / \sqrt{2}$. The three edges starting from the vertex at the origin are characterized by the unit vectors $-\mathbf{e}_{2}$ and $\left(\mathbf{e}_{2} \pm \sqrt{3 / 2}\left(\mathbf{e}_{1}+\mathbf{e}_{3}\right)\right) / 2$. The corner angles 
of a patch are $90^{\circ}, 90^{\circ}, 90^{\circ}, 60^{\circ}$, and hence the defect is $\delta=1$. We have $V_{\mathrm{OC}}^{\mathrm{R}}=e^{3} / 54$ and $V_{\mathrm{WC}}^{\mathrm{R}}=\infty$. The characteristic length $e$ is varied during the computation.

\section{THE NUMERIC SCHEME}

The following constructions are visualized in Fig. (22). We choose $r$ and $s$ as integers and endow the rectangle $(0 \leq u \leq r, 0 \leq v \leq s)$ with $(r+1) \times(s+1)$ grid points at $(u=i, v=j) \quad(i=0,1,2 \ldots r, j=0,1,2 \ldots s)$. The function $\theta(u, v)$ which generates the surface is replaced by discrete values $\theta_{i j}$ at these grid points. The position vectors at the grid points are

$$
\mathbf{x}_{i j}=\left(\mathbf{x}^{\mathrm{R}}+\zeta \mathbf{m}\right)(u=i, v=j)=e\left(\tilde{\mathbf{x}}_{i j}^{\mathrm{R}}+\theta_{i j} \mathbf{m}_{i j}\right)
$$

We also construct three kinds of points which we call nodes. The $r \times s$ nodes of the first kind lie in the centre of four neighbouring grid points, i.e. at $(u=i-1 / 2, v=j-1 / 2) \quad(i=1,2 \ldots r, j=1,2 \ldots s) . \quad$ The $2(r+s)$ nodes of the second kind lie in the centre of two neighbouring grid points on a boundary, i.e. at $(u=i-1 / 2, v=0$ or $v=s) \quad(i=1,2 \ldots r) \quad$ or $\quad$ at $(u=0$ or $u=r, v=j-1 / 2)(j=1,2 \ldots s)$.

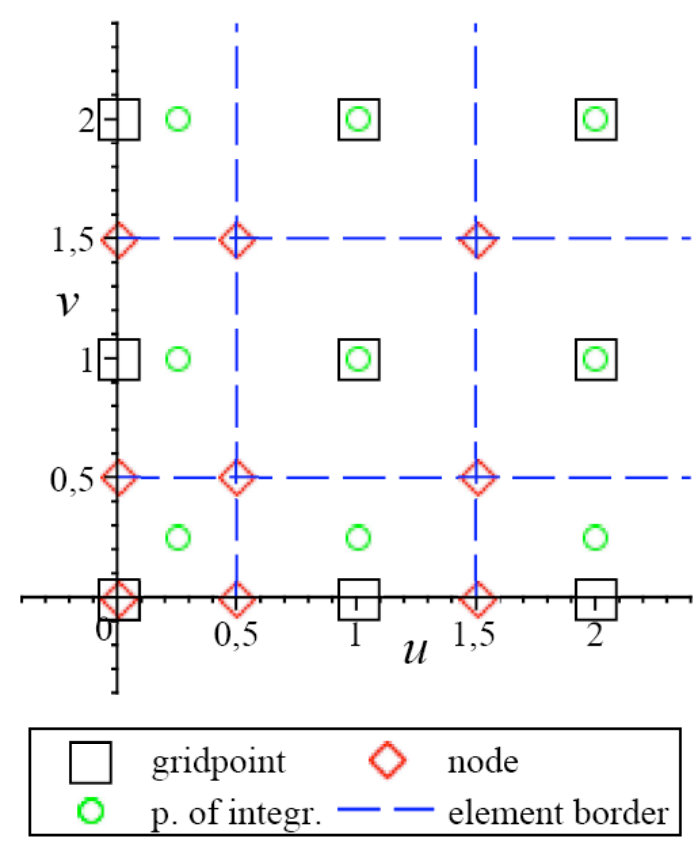

Fig. (22). Construction of nodes and elements.

The four nodes of the third kind coincide with the grid points at the four corners. Any four neighbouring nodes define a region which we call an element. There are $(r-1) \times(s-1) \quad$ square elements of area $1,2(r+s-2)$ rectangular elements of area $1 / 2$ and four square elements of area $1 / 4$.

Next we need the tangential planes at the nodes. If we interpolate the values of $\mathbf{x}$ at four neighbouring grid points by a complete quadratic expression, then the tangential vectors at the appertaining node of the first kind read

$$
\begin{aligned}
& \mathbf{g}_{u i+1 j+1}=\mathbf{x},_{u}(u=i+1 / 2, v=j+1 / 2) \\
& =\frac{1}{2}\left(\mathbf{x}_{i+1 j+1}-\mathbf{x}_{i j}+\mathbf{x}_{i+1 j}-\mathbf{x}_{i j+1}\right) \\
& \mathbf{g}_{v i+1 j+1}=\mathbf{x},_{v}(u=i+1 / 2, v=j+1 / 2) \\
& =\frac{1}{2}\left(\mathbf{x}_{i+1 j+1}-\mathbf{x}_{i j}-\mathbf{x}_{i+1 j}+\mathbf{x}_{i j+1}\right) \\
& (i=0,1,2 \ldots r-1, j=0,1,2 \ldots s-1) . \quad \text { They allow the }
\end{aligned}
$$
computation of $\mathbf{w}, \psi, \mathbf{n}, \mathbf{g}^{\mathrm{u}}$, and $\mathbf{g}^{v}$. The tangential plane at a node of the second or third kind is obtained in a different manner. We start from $\mathbf{x}=\mathbf{x}^{\mathrm{R}}+\zeta \mathbf{m}$ and find

$$
\begin{aligned}
& \mathbf{g}_{u}=\mathbf{x},{ }_{u}=\tilde{\mathbf{g}}_{u}+\zeta{ }_{u} \mathbf{m} \quad \text { with } \quad \tilde{\mathbf{g}}_{u}=\mathbf{x}^{\mathrm{R}}{ }_{{ }_{u}}+\zeta \mathbf{m},_{u} \\
& \mathbf{g}_{v}=\mathbf{x},{ }_{v}=\tilde{\mathbf{g}}_{v}+\zeta{ }_{, v} \mathbf{m} \quad \text { with } \quad \tilde{\mathbf{g}}_{v}=\mathbf{x}^{\mathrm{R}},_{v}+\zeta \mathbf{m},{ }_{v}
\end{aligned}
$$

The partial derivatives of $\mathbf{x}^{\mathrm{R}}$ and $\mathbf{m}$ at the nodes can be gained from the analytical expressions (20) to (22).

First considering the nodes of the third kind, we observe that the tangential plane at any corner of our reference patch is orthogonal to each of the two intersecting planes of symmetry so that the normal vector $\mathbf{n}$ must coincide with the director $\mathbf{m}$. Because of

$$
\mathbf{n}=\psi \mathbf{w}=\mathbf{m}
$$

we infer the values of $\psi$ and $\mathbf{w}$ from

$$
\frac{1}{\psi}=\mathbf{w} \cdot \mathbf{m}=\left(\mathbf{g}_{u} \times \mathbf{g}_{v}\right) \cdot \mathbf{m}=\left(\tilde{\mathbf{g}}_{u} \times \tilde{\mathbf{g}}_{v}\right) \cdot \mathbf{m}
$$

and

$$
\mathbf{w}=\frac{1}{\psi} \mathbf{m}
$$

and also obtain

$$
\begin{aligned}
& \mathbf{g}^{u}=\psi^{2} \mathbf{g}_{v} \times \mathbf{w}=\psi \tilde{\mathbf{g}}_{v} \times \mathbf{m} \\
& \mathbf{g}^{v}=\psi^{2} \mathbf{w} \times \mathbf{g}_{u}=-\psi \tilde{\mathbf{g}}_{u} \times \mathbf{m}
\end{aligned}
$$

Next, we discuss nodes of the second kind and select the boundary with $v=0$. The outer unit normal vector of the symmetry plane there shall be denoted by e. Making use of

$$
\begin{aligned}
& \mathbf{e} \times \mathbf{w}=\mathbf{e} \times\left(\mathbf{g}_{u} \times \mathbf{g}_{v}\right)=\left(\mathbf{e} \cdot \mathbf{g}_{v}\right) \mathbf{g}_{u}-\left(\mathbf{e} \cdot \mathbf{g}_{u}\right) \mathbf{g}_{v} \\
& =\left(\mathbf{e} \cdot \tilde{\mathbf{g}}_{v}+\zeta,{ }_{v} \underline{\mathbf{e} \cdot \mathbf{m}}\right) \mathbf{g}_{u}-\underline{\left(\mathbf{e} \cdot \mathbf{g}_{u}\right)} \mathbf{g}_{v}
\end{aligned}
$$

we find the representation

$$
\begin{aligned}
& \mathbf{w}=(\mathbf{e} \cdot \mathbf{e}) \mathbf{w}-\underline{(\mathbf{e} \cdot \mathbf{w})} \mathbf{e} \\
& =(\mathbf{e} \times \mathbf{w}) \times \mathbf{e}=\left(\mathbf{e} \cdot \tilde{\mathbf{g}}_{v}\right)\left(\mathbf{g}_{u} \times \mathbf{e}\right)
\end{aligned}
$$


The underlined terms vanish since the vectors $\mathbf{m}, \mathbf{g}_{u}$, and $\mathbf{w}$ lie altogether in the plane of symmetry and are therefore normal to $\mathbf{e}$.

We obtain $\mathbf{g}_{u}$ at the node by applying quadratic interpolation of $\mathbf{x}$ and find

$$
\mathbf{g}_{u i+10}=\mathbf{x},{ }_{u}(u=i+1 / 2, v=0)=\mathbf{x}_{i+10}-\mathbf{x}_{i 0}
$$

while linear interpolation of $\zeta$ yields the value

$$
\zeta(u=i+1 / 2, v=0)=\frac{1}{2}\left(\zeta_{i+10}+\zeta_{i 0}\right)
$$

which is needed in the computation of $\tilde{\mathbf{g}}_{v}$. The value of $\psi$ can be read from

$$
\frac{1}{\psi^{2}}=\mathbf{w} \cdot \mathbf{w}=\left(\mathbf{e} \cdot \tilde{\mathbf{g}}_{v}\right)^{2}\left(\mathbf{g}_{u} \cdot \mathbf{g}_{u}\right)=\left(\mathbf{e} \cdot \tilde{\mathbf{g}}_{v}\right)^{2} g_{u u}
$$

and the cotangent vectors are

$$
\mathbf{g}^{v}=\psi^{2} \mathbf{w} \times \mathbf{g}_{u}=\frac{1}{\mathbf{e} \cdot \tilde{\mathbf{g}}_{v}} \mathbf{e}
$$

and

$$
\begin{aligned}
& \mathbf{g}^{u}=\psi^{2} \mathbf{g}_{v} \times \mathbf{w}=\psi^{2}\left(\mathbf{e} \cdot \tilde{\mathbf{g}}_{v}\right) \mathbf{g}_{v} \times\left(\mathbf{g}_{u} \times \mathbf{e}\right) \\
& =\frac{1}{g_{u u}}\left(\mathbf{g}_{u}-\frac{g_{u v}}{\mathbf{e} \cdot \tilde{\mathbf{g}}_{v}} \mathbf{e}\right)
\end{aligned}
$$

The latter requires the knowledge of

$$
g_{u v}=\mathbf{g}_{u} \cdot \mathbf{g}_{v}=\mathbf{g}_{u} \cdot \tilde{\mathbf{g}}_{v}+\zeta, \mathbf{g}_{u} \cdot \mathbf{m}
$$

But the value of $\zeta{ }_{,}$can be inferred from the statement

$$
0=\mathbf{w} \cdot \mathbf{g}_{v}=\mathbf{w} \cdot\left(\tilde{\mathbf{g}}_{v}+\zeta,{ }_{v} \mathbf{m}\right)
$$

and reads

$$
\zeta,_{v}=-\frac{\mathbf{w} \cdot \tilde{\mathbf{g}}_{v}}{\mathbf{w} \cdot \mathbf{m}}=-\frac{\left(\mathbf{g}_{u} \times \mathbf{e}\right) \cdot \tilde{\mathbf{g}}_{v}}{\left(\mathbf{g}_{u} \times \mathbf{e}\right) \cdot \mathbf{m}}
$$

At last, we are interested in numerical integration. The integral of a function $\phi$ on our surface patch is computed as a sum of integrals over the $(r+1) \times(s+1)$ elements in the $u, v$-plane. The numerical evaluation of an integral over one element uses one point of integration, located in the centre. Noting (13), we arrive at

$$
\int_{A_{\mathrm{C}}} \phi d A=\int_{u=0}^{r} \int_{v=0}^{s} \frac{\phi}{\psi} d u d v \approx \sum_{k} \frac{\phi_{k}}{\psi_{k}} \mathrm{v}_{k}
$$

The weight of the element with number $k$ is

$$
v_{k}=d_{u k} d_{v k}
$$

where $d_{u k}$ and $d_{v k}$ denote the edge lengths of the element in the directions $u$ and $v$, respectively, which are either 1 or $1 / 2$.
Some of our functions $\phi$ depend on the curvature invariants. So we need the mixed components of the curvature tensor $\mathbf{C}$ at the points of integration. They are

$$
\begin{aligned}
& c_{\beta}^{\alpha}=\mathbf{g}^{\alpha} \cdot \mathbf{C} \cdot \mathbf{g}_{\beta}=-\mathbf{g}^{\alpha} \cdot \mathbf{n} \otimes \nabla_{T} \cdot \mathbf{g}_{\beta}=-\mathbf{g}^{\alpha} \cdot \mathbf{n},{ }_{\beta} \\
& =-\psi \mathbf{g}^{\alpha} \cdot \mathbf{w},{ }_{\beta}-\underline{\mathbf{g}^{\alpha} \cdot \mathbf{w}} \psi,_{\beta} \quad \alpha, \beta=u, v
\end{aligned}
$$

where the underlined term vanishes. The curvature invariants read

$$
\operatorname{tr} \mathbf{C}=c_{u}^{u}+c_{v}^{v} \quad \operatorname{det} \mathbf{C}=c_{u}^{u} c_{v}^{v}-c_{v}^{u} c_{u}^{v}
$$

The values of $\psi$ and $\mathbf{g}^{\alpha}$ at the point of integration are taken as the mean of the values of the adjacent nodes, which complies with bilinear interpolation. We also need the partial derivatives of $\mathbf{w}$. If we interpolate the values of $\mathbf{w}$ at four neighbouring nodes by a complete quadratic expression, then we get, at the point of integration,

$$
\begin{aligned}
& \mathbf{w}_{,_{i j} j}=\frac{1}{2 d_{u}}\left(\mathbf{w}_{i+1 j+1}-\mathbf{w}_{i j}+\mathbf{w}_{i+1 j}-\mathbf{w}_{i j+1}\right) \\
& \mathbf{w}_{,_{i j}}=\frac{1}{2 d_{v}}\left(\mathbf{w}_{i+1 j+1}-\mathbf{w}_{i j}-\mathbf{w}_{i+1 j}+\mathbf{w}_{i j+1}\right)
\end{aligned}
$$

Finally, we consider integrals which yield volumes. The volume of the oil-sided and water-sided cavities within a cell is given by

$$
\begin{aligned}
& V_{\mathrm{O} / W C}=V_{\mathrm{O} / W C}^{\mathrm{R}} \mp V^{\mathrm{D}} \\
& -\int_{A_{\mathrm{C}}}\left(\frac{h}{2} \mp \operatorname{tr} \mathbf{C} \frac{h^{2}}{8}+\operatorname{det} \mathbf{C} \frac{h^{3}}{24}\right) d A
\end{aligned}
$$

Here $V_{\mathrm{O} / W C}^{\mathrm{R}}$ denotes those parts of the cell volume which lie on the oil side and the water side of the reference patch, $V^{\mathrm{D}}$ is the volume between the plane reference patch and the curved surface patch, while the last integral describes that part of the film volume which is situated on the oil side and the water side of the middle surface, respectively. If the displacement $\hat{\zeta}$ increases from 0 to the final value $\zeta$ according to the law

$$
\hat{\zeta}=\zeta \frac{1+t}{2}, \quad-1 \leq t \leq 1
$$

then the oil-sided volume decrease is given by the covered volume

$$
\begin{aligned}
V^{\mathrm{D}}= & \int_{u=0}^{r} \int_{v=0}^{s} \int_{\hat{\zeta}=0}^{\zeta} \mathbf{x},{ }_{u} d u \times \mathbf{x},{ }_{v} d v \cdot \mathbf{x}, \hat{\zeta} d \hat{\zeta} \\
= & \int_{u=0}^{r} \int_{v=0}^{s} \int_{t=-1}^{1}\left(\mathbf{x}^{\mathrm{R}}{ }_{u}+\left(\zeta,{ }_{u} \mathbf{m}+\zeta \mathbf{m},{ }_{u}\right) \frac{1+t}{2}\right) \times \\
& \left(\mathbf{x}^{\mathrm{R}},{ }_{v}+\left(\zeta,{ }_{v} \mathbf{m}+\zeta \mathbf{m},{ }_{v}\right) \frac{1+t}{2}\right) \cdot \mathbf{m} \frac{\zeta}{2} d t d u d v
\end{aligned}
$$




$$
\begin{gathered}
=\int_{u=0}^{r} \int_{v=0}^{s}\left(\left(\mathbf{x}^{\mathrm{R}},{ }_{u}+\frac{1}{2} \zeta \mathbf{m},{ }_{u}\right) \times\left(\mathbf{x}^{\mathrm{R}},{ }_{v}+\frac{1}{2} \zeta \mathbf{m},{ }_{v}\right) \zeta\right. \\
\left.+\frac{1}{12} \mathbf{m},{ }_{u} \times \mathbf{m},{ }_{v} \zeta^{3}\right) \cdot \mathbf{m} d u d v \\
\approx \sum_{k}\left(\left(\mathbf{x}^{\mathrm{R}},{ }_{u}+\frac{1}{2} \zeta \mathbf{m},{ }_{u}\right) \times\left(\mathbf{x}^{\mathrm{R}},{ }_{v}+\frac{1}{2} \zeta \mathbf{m},{ }_{v}\right) \zeta\right. \\
\left.+\frac{1}{12} \mathbf{m},{ }_{u} \times \mathbf{m},{ }_{v} \zeta^{3}\right)\left.\right|_{k} \cdot \mathbf{m}_{k} v_{k}
\end{gathered}
$$

[5] Tlusty T, Safran SA, Strey R. Topology, phase instabilities, and

The values of $\mathbf{x}^{\mathrm{R}}, \mathbf{m}$ and their partial derivatives at the point of integration are obtained from the analytical expressions (20) to (22). The value of $\zeta$ is obvious if the point of integration coincides with a grid point, otherwise it is generated by linear or bilinear interpolation of the values at two or four neighbouring grid points.

\section{REFERENCES}

[1] Strey R. Microemulsion microstructure and interfacial curvature. Colloid Polym Sci 1994; 272: 1005-1019.

[2] Gompper G, Schick M. Self-assembling amphiphilic systems, phase transitions. London: Academic Press 1994; vol. 16.

[3] Safran SA. Microemulsions: An ensemble of fluctuating interfaces. In: Chen S-H, Huang JS, Tartaglia P, Eds. Structure and dynamics Wetting of microemulsion networks. Phys Rev Lett 2000; 84: 1244-7.

[6] Schwarz HA. Gesammelte Mathematische Abhandlungen. Springer 1890 .

[7] Anderson DM, Davis HT, Nitsche JCC, Scriven LE. Periodic surfaces of prescribed mean curvature. Adv Chem Phys 1990; 77 337-96.

[8] Schwarz US, Gompper G. Stability of bicontinuous cubic phases in ternary amphiphilic systems with spontaneous curvature. J Chem Phys 2000; 112 (8): 3792-802.

of strongly interacting colloids and supramolecular aggregates in solution. Dordrecht: Kluwer 1992; pp. 237-263.

Safran SA. Curvature elasticity of thin films. Adv Phys 1999; 48 (4): $395-448$

9] Leitão H, Somoza AM, Telo da Gama MM, Sottmann T, Strey R. Scaling of the interfacial tension of microemulsions: a phenomenological description. J Chem Phys 1996; 105 (7): 2875-83.

[10] Lade O, Krawietz A. A bending elasticity approach to the threephase coexistence of microemulsions. J Chem Phys 2001; 115 (23): 10986-97.

[11] Krawietz A. Non-Linear Continuum mechanics of microemulsions and amphiphilic monolayers. Pro BUSINESS: Berlin 2007 (can also be found on [14]).

[12] Flügge W. Stresses in shells. Springer: Berlin 1960.

[13] Helfrich W. Elastic properties of lipid bilayers: theory and possible experiments. Z Naturforsch 1973; C 28: 693-703.

[14] Available at: krawietz.homepage.t-online.de/equilibrium.html

[15] Leach AR. Molecular Modelling. Prentice Hall: Harlow 2001.

[16] Robledo A, Varea C. Can the Helfrich free energy for curved interfaces be derived from first pinciples? Phy A 1996; 231: 178-90.

[17] Zhou S. Formally 'exact' first-order Taylor series expansion for density functional theory. N J Phys 2002; 4, 36.

(c) Arnold Krawietz; Licensee Bentham Open.

This is an open access article licensed under the terms of the Creative Commons Attribution Non-Commercial License (http://creativecommons.org/licenses/by-nc/3.0/) which permits unrestricted, non-commercial use, distribution and reproduction in any medium, provided the work is properly cited. 